\title{
1 Ordination analysis in sedimentology, geochemistry 2 and paleoenvironment - background, current trends 3 and recommendations
}

4

Or M. Bialik ${ }^{1, *}$, Emilia Jarochowska ${ }^{2, *}$, Michal Grossowicz ${ }^{3}$

1. Marine Geology and Seafloor Surveying, Department of Geosciences, University of Malta, Msida MSD 2080, Malta.

2. GeoZentrum Nordbayern, Friedrich-Alexander-Universität Erlangen-Nürnberg, 91054 Erlangen, Germany.

3. GEOMAR Helmholtz Centre for Ocean Research Kiel, 24105 Kiel, Germany.

*corresponding authors: or.bialik@um.edu.mt (OMB); emilia.jarochowska@fau.de (EJ)

This document is a non-peer reviewed preprint submitted to EarthArXiv. It is under review in The Depositional Record. The authors can be reached via Twitter under @BialikOr, @Emiliagnathus and @micgros

\section{Abstract}

Ordination is the name given to a group of methods used to analyze multiple variables without preceding hypotheses. Over the last few decades the use of these methods in Earth science in general, and notably in analyses of sedimentary sources, has dramatically increased. However, with limited resources oriented towards Earth scientists on the topic, the application of ordination analysis is at times suboptimal and misuse by authors can occur. This text was written for researchers with little to no experience with ordination with the aim of exposing them to the utility and the pitfalls of this branch of exploratory statistics. To do so, we offer a detailed review of three ordination methods: principal component analysis (PCA), non-metric multidimensional scaling (NMDS) and detrended correspondence analysis (DCA). We then present a survey of 163 publications in Earth science, in which these ordination methods were used. We summarize how, why and on what type of data ordination was used and outline common mistakes and misuses identified in those publications. Notably, we found issues with reproducibility, documentation, data set dimensions and transformations. Based on this survey, we offer a recommended workflow for Earth scientists who wish to apply ordination. Additionally, this article is accompanied by highly annotated $\mathrm{R}$ scripts for novice users to use these methods. 
35 Keywords: PCA, NMDS, DCA, exploratory statistics, clustering, dimension reduction, multivariate statistics

\section{1. Introduction}

38 Geology, as a science, combines descriptive (qualitative and semiquantitative) and quantitative approaches. As the discipline evolved the lexical richness of descriptive tools had increased, whereas technological advances have provided geoscientists with a wide range of quantitative tools. This allows modern geoscientists to generate an incredible richness of data. For example, a study may include now textural description, elemental chemistry, isotopes and mineralogy from tens or even hundreds of samples. To deal with these progressively larger data sets, researchers have been turning to multivariate analysis methods, and particularly to ordination.

Geoscientists can approach this data in an exploratory fashion, a hypothesis testing one or a combination of both. However, even when testing hypotheses, geoscientists usually look for positive evidence. In that sense, geology and it's sister disciplines do not adhere to the Popper school of testing scientific theories through falsifiability (Popper, 1934). Authors such as Birks (1985) argued that geology was/is still in the empirical-descriptive or narrative phase of its development and would develop onwards to an analytical phase governed by the hypotheticodeductive approach. While geosciences have indeed developed in this direction, it is a field that still has a very strong exploratory component. This, in turn, requires a different statistical process then that implemented in disciplines driven more by falsifiability (Mulaik, 1985). Ordination has been used in Earth sciences for many years, with the earliest mention of it dating back to the 1940s (Griffiths, 1947), but its use has proliferated over the last thirty years as personal computing and statistical programs became more prevalent. Unfortunately, most popular and widely accessible resources on multivariate statistics are oriented towards statisticians, ecologists, or social scientists, and not geoscientists. For example, at the time of the writing of this manuscript, of the 118 books on multivariate statistics available in the Springer catalogue only two are specified for geoscientists (Brown, 1998; Wackernagel, 2003), and only one of them discusses ordination methods. A similar underrepresentation is also observed in the Elsevier and Blackwell catalogues. This highly limits an Earth science student or scientist interested in applying these methods as the translation of terms and examples from these fields into geoscience might often be non-trivial. As a result, multivariate statistics in general, and ordination in particular, are not only less accessible to Earth scientists, they are also farther removed from their intuitive toolbox. Most ordination techniques have been 
designed to handle species abundance or occurrence matrices (Hammer and Harper, 2006; Oksanen et al., 2019), which require different transformations techniques and choice of parameters. This results in useful statistical tools not being in the forethought of Earth scientists when conducting research and compiling manuscripts. Moreover, as will be discussed later on in the text, when these methods are implemented, mistakes and misuses are likely to occur.

An additional concern regarding the use of statistical tools has to do with reproducibility. Over the last few years a growing concern in the scientific community has risen about the reproducibility of scientific results (Nissen et al., 2016; Cooper, 2018). Implementation affects reproducibility as different statistical packages and even slightly different workflows can result in different outcomes. Proper reporting of the assumptions, what analysis methods were used and why, and their output is considered crucial for comparability between studies (Amrhein, Trafimow and Greenland, 2019). This raises the need for better reporting and documentation of the statistical methods used and deeper understanding of their underlying algorithm.

There is a need to have a discussion about the implementation of those statistical tools to be more suitable to sedimentology, geochemistry, paleoenvironmental studies et hoc genus omne. Since, as noted, these fields often have a strong exploratory nature, here we set out to examine the use of ordination in these fields. In this study we present a review on ordinations, and describe in detail three selected popular methods: principal component analysis (PCA), non-metric multidimensional scaling (NMDS) and detrended correspondence analysis (DCA). The math and equations are not detailed here, instead we provide carefully selected references on where to find them in an approachable form. We here provide a roadmap of sources which we find particularly useful for geoscientists. We then present a survey of the use of these ordination methods in Earth Sciences focusing on work done on rock, water, sediment and fossils. From this review, we identify several common mistakes and issues with the application of ordination methods and interpretation of the results. We conclude by suggesting several recommended workflows and ways to avoid the pitfalls we identified.

\subsection{What is ordination?}

Ordination is the name for a family of multivariate analysis methods for exploratory data analysis (Gower, 1987). The common thread of these techniques is that they all order multivariate objects in a fashion that places similar objects near each other with dissimilar objects further away. The term exploratory data analysis here refers not so much to dealing with unknown settings for the first time but rather an approach to analyzing data sets by summarizing their main characteristics and leading to formulation of hypotheses rather than testing them (Chatfield, 1995). This approach falls into the broader category of machine 
learning without supervision (Hastie, Tibshirani and Friedman, 2009). As such, ordination methods were principally developed to allow a researcher to examine a data set tabula rasa and identify the relations in it.

In our survey, ordination was used for three principal goals: 1) to understand or identify the relation between variables; 2) to differentiate or cluster data points; and 3) generate indices. The first two are clear derivatives of the exploratory nature of ordination methods. The third is a utilization of the property of most ordination methods - dimension reduction. Ordination methods represent a higher number of dimensions by a smaller number of dimensions called components (Gauch and Whittaker, 1972; Syms, 2008). This can allow the representation of multiple variables as a much smaller number (one to three) of variables (indices) which should represent the highest proportion of the variability that can be shown in this reduced number of dimensions. Sedimentological data seta often consist of proxies, e.g. for the redox conditions, for distance from sediment source etc., where multiple variables are driven by a common underlying one, but recording it with different sensitivities and measured with different error terms. In dealing with proxy data, reduction of dimensions typically aims at reconstructing this underlying variable through a combination of co-varying proxy variables through which this variable is approximated (e.g., Gupta and Thomas, 1999; Abdelhady and Fürsich, 2014; Bitušík et al., 2018; Koutsodendris et al., 2020; see Table S2).

There are multiple ordination methods developed over the course of the 20th century, from the classical canonical correlation (Austin, 1968) and polar ordination (Bray and Curtis, 1957) to more modern methods such as canonical correspondence analysis (ter Braak, 1986) and redundancy analysis (ter Braak and Prentice, 1988). Here we focus and discuss three of them: PCA, NMDS and DCA, summarized in Table 1. These methods are commonly in use across multiple disciplines including Earth sciences. They are implemented in all major statistical packages and described in approachable textbooks. Each ordination method is a class of algorithms in its own right with a common approach. This means that the same method might produce different outputs between two software packages due to differences in the underlying algorithms they use.

\subsection{Similarity Indices}

An underlying aspect of ordination is the measurement of how similar or dissimilar data points are from one another. The use of similarity indices (also called similarity measures or similarity functions) is a means to evaluate this between samples. Different ordination methods use as input distance matrices, which describe the distances between each point and all the other points in the data set. However, similarity indices can also be used on their own. There is a large variety of similarity indices, Sneath and Sokal (1973) and Johnston (1976) listed over 
139 thirty different indices and offer an extensive review of them. Some indices summarize the

140 values of all variables into a single index (community structure measures sensu Pinkham and 141 Pearson, 1976), whereas other pair attributes.

142 An important point to remember with regards to similarity indices are their underlying 143 assumptions and methods. For example, while the input data to the index might be in 144 absolutes, the index might treat them as proportions, effectively turning an open data set into 145 a closed one (Hammer and Harper, 2007, also see below), that is to say defined as part of a 146 sum, which has a knock-on effect on the distributions of the variables. Many indices 147 implemented in popular packages have been explicitly developed to handle biological diversity 148 aspects and are derived from common ways of measuring diversity (see Jost et al., 2011, for 149 a detailed analysis of the properties of common indices), and therefore many of them will not 150 be relevant for sedimentological data.

151 Three indices that should be mentioned here, as they are relevant to the text, are Jaccard, 152 Bray-Curtis and Gower. The Jaccard index (Jaccard, 1908) is one of the oldest indices still in 153 use. This method specifically was designed for binary (also called presence/absence or PA) 154 variable sets by using the size of the intersection divided by the size of the union of the sample 155 sets. Another commonly used index is the Bray-Curtis dissimilarity index (Bray and Curtis, 156 1957), this index has been specifically named as one of the more robust indices for 157 communities (Bloom, 1981). This index is designed for compositional data sets which follow a 158 consistent sampling protocol (e.g. the same number of points is counted in multiple thin 159 sections; same number of foraminifera counted in each sample), and is computed based on 160 the ratio of lesser values to the sum of the vectors. Nonetheless, data sets need to be prepared 161 in consideration of the properties of this index. The index approaches 0 when sample sizes 162 are very different, regardless how similar their composition is. In such cases, samples need to 163 be standardized to be used with this index, e.g. in the number of counts in a sample can be 164 standardized to correspond to proportions in sample; or a different index may be more 165 appropriate. For a detailed illustration of the limitations of the Bray-Curtis index and possible 166 solutions, see Jost et al. (2011) and Chao et al. (2006). Gower's similarity coefficient (Gower, 167 1971; Podani, 1999) is a notably important index as it is one of the more suitable for mixed 168 data (van de Velden et al., 2019). This index uses a weight function to compute the similarity 169 and eliminate objects equal to zero. 
170

171

172

173

174

175

176

177

178

179

180

181

182

183

184

185

186

187

188

189

190

191

192

193

\subsection{Closed and open data sets, compositional and non-}

\section{compositional data}

The mathematical meaning of open and closed sets are complex topological terms defining sets of points meeting specific conditions in a topological space. Here we refer to a simpler case (Reyment and Savazzi, 1999) where the data matrix is constrained (or closed) or unconstrained (open). Closed data sets are also known as compositional. Egozcue (2009) provided a handy definition from these as "compositional data thus quantitatively expresses relative contributions of variables under consideration of a certain whole, which carry relative information between the components". This is sometimes defined in more simplified terms as compositional data being a positive value multivariate data that sum up to a constant, with an underlying notion that a change in one value of a given variable will permeate changes across the entire variables. This interdependence is very well illustrated in data given in percent. If in an assemblage there is a set number of elements summed and divided to percent of the total, and on a reanalysis one of values of this assemblage changes - all others will change to reach a sum of $100 \%$.

Compositional (closed) and non-compositional (open) data sets do not behave the same way and have different geometrical properties (Aitchison, 1982; Filzmoser, Hron and Reimann, 2009; Greenacre, 2018). As a result, the distributions of compositional data sets are different from those of non-compositional data sets (where the variables do not have interdependence). This requires that compositional data sets will be pre-treated if one wishes to apply methodologies designed for non-compositional data sets or that makes assumptions about the underlying probability distribution (parametric methods), assumed for non-compositional data sets.

\section{Ordination methods}

\subsection{PCA}

Principal component analysis (PCA) is an approach for finding variables (referred to as components) that account for the maximum amount of the variance in a multidimensional data set (Hotelling, 1933; Duneman, 1989). These components are linear combinations of the original variables. By summing the total variation of all components and defining the variation of each component by that number, we obtain the percent of explained variance on that component. The variances of each component are often presented in a form called a "scree plot". The method required input must be metric, that is to say, the input cannot be boolean 
202

203

204

205

206

207

208

209

210

211

212

213

214

215

216

217

218

219

220

221

222

223

224

225

226

227

228

229

230

231

232

233

234

235

236

237

238

nor can it be made of distinct categories (which are often used to describe sediments and rocks, e.g. facies and lithology) nor can it be ranked (see section 4.2 for a classification of levels of measurement). Here we refrain from repeating the description of mathematical operations behind PCA, but interested readers can find approachable explanations in the textbooks such as Rencher (2003) or Gauch (1982).

In a bare-bone algorithm, based only on variance, units are very important for two reasons. First, the size of numbers in each variable will affect the calculation of the sum of squared distances, emphasising large numbers. For example, if all variables are given in the same unit, and there are orders of magnitude of difference between variables (e.g. micrite in a point count of thin sections from a mudstone), the larger numbers will dominate the axis of maximal variance (referred to as PC1). This is by design, PCA is optimized to represent the maximum variability and not nuances of small sample variance. Secondly, any unit that is given as a fraction of a whole, e.g. percent weight, $\mathrm{mg} / \mathrm{kg}$, ppm etc. may lead to a closed-sum problem, which can compromise linear relationships between variables due to a poor representation of the dimensional centre and distribution around it (Filzmoser, Hron and Reimann, 2009; Tolosana-Delgado, 2012).

PCA relies on the matrix of covariance between each pair of variables in the data set. Covariances are, however, not meaningful if variables are measured in different units. A more complex, but not unimaginable in sedimentology, would be if the variables are measured in the same units but are not measured on a ratio scale, as would be e.g. for frequencies in bins, especially if bins are defined differently for two variables, or for ratio variables, which are unitless. Some of such cases may be hard to identify, but as a rule of thumb we recommend that if variables are not clearly measured in the same units, the matrix of covariance should not be used. Instead, PCA can be calculated using the matrix of correlations between variables. This is not the default option in most packages and needs to be consciously chosen by the user.

PCA was originally designed with data sampled from a multivariate normal distribution in mind. This means that, ideally, the variables should have a multinormal distribution or be normalized (Legendre and Legendre, 2012). Performance of PCA does not require variables to be normalized (see section 2.5) or have a normal distribution, and deviation from normality might not bias the analysis (Ibanez, 1971). However, strong deviations from symmetry - which is a property of the normal distribution - will affect the performance of PCA negatively (Hammer and Harper, 2007). In the absence of symmetry in the distributions, the resulting ordination might be very sensitive to small changes. A common approach is to apply a monotone transformation that reduces the skewness (asymmetry) of the distribution. We discuss the topic of transformation farther along the text, for more details both Legendre and Legendre (2012) and Filzmoser et al. (2009) offer overviews of available transformation methods, the 
239 later being more oriented towards Earth sciences. PCA also suffers from several other 240 sensitivity issues related to the data distribution (Shi, 1993), for some of which DCA and NMDS 241 were specifically designed to overcome.

\section{2.2. NMDS}

244 Non-metric multidimensional scaling (NMDS, sometimes nMDS, NMS, or MDS) is an indirect 245 gradient analysis approach which produces an ordination (Kruskal, 1964). However, rather 246 than using some metric of distance, NMDS substitutes the original distance data with ranks.

247 The use of ranks omits some of the issues associated with using absolute distance (e.g. 248 sensitivity to transformation), and as a result, is a much more flexible technique that accepts 249 a variety of types of data (Field, Clarke and Warwick, 1982), it is also one of the best predictive 250 ordination methods in general (Wildi, 2018). NMDS operates as an iterative process: first, it generates a dissimilarity matrix for every pair of samples, for example by using the Bray-Curtis dissimilarity index. The NMDS algorithm then finds an optimal monotonic transformation of the similarities in order to obtain optimally scaled data, and tests that versus the similarity matrix. It then rearranges the configurations in order to minimize the stress - the difference between the original ranked distances and those in the transformed output. The algorithm repeats these steps until the stress is reduced to a predefined level. Ideally, the stress should be less than 0.05 although stress of $0.05-0.10$ is still good, although there is a risk of false inferences. That said, a very large number of samples could lead to high stress despite reasonable inference (Dexter, Rollwagen-Bollens and Bollens, 2018). This iterative nature of NMDS makes it computationally heavy (Alotaibi, Rayward-Smith and de la Iglesia, 2011) which could be an issue with very large data sets, although more modern algorithms have improved computation time (Taguchi and Oono, 2005). Additionally, this means that NMDS is expected to return the similar but not identical result on different runs (Hammer and Harper, 2007). This last property is particularly important to note when using NMDS to generate indices. Even if data is not changed, different permutations could result in slightly different results, which in turn could decrease the signal-to-noise ratio. This can be circumvented by seeding the random number generator by a fixed number (see 3.3.2.4).

269 Detrended correspondence analysis (DCA) is a derivative of the correspondence analysis (CA) method (Hill, 1979). While CA operates very similarly to PCA, it determines the 
272 rather than the variance. DCA adds another layer of operation aimed at neutralizing the "arch

273 effect" CA suffers from, where the points forming a gradient reconstructed along the second 274 axis are distributed along an arch relative to the first axis. This arch results in 275 misrepresentation of the gradient orthogonal to the first gradient, but is a conflation of both 276 gradients. This detrending can be done in two methods, either by expressing each subsequent 277 axis as a polynomial function of the prior axis or by a segmentation method in which segments 278 of each axis are centred to have a zero mean relative to the subsequent axis. Following this 279 step, a nonlinear rescaling is implemented to shift sample scores along each axis so that the 280 average width would become 1 . These distortions of the axis, especially with the segmentation 281 method, is the core a critic of DCA (Wartenberg, Ferson and Rohlf, 1987) arguing that it masks, 282 through mathematical manipulations, the data's real curvilinear structure and as such hinders 283 the understanding of the real data and identification of the causes of the observed distribution.

\subsection{Clustering and cluster testing}

285 Clustering is a different subset of exploratory techniques for identifying groups and subgroups in a multivariate data set, in order words - identify which objects in a data set are similar to each other and to what level (Romesburg, 2004). Ordination methods are capable of detecting groups (clusters) if they are present in the data set, but they are not clustering methods. Paired with their relation to the variables, it is possible to formulate hypotheses about distribution patterns in the data. The clusters might be a priori, defined for example by lithology or temporal position and reinforced by the ordination. Alternatively, the clusters might be defined a posteriori (learning without supervision) to the ordination based on the inferred distribution of samples or variables across the main components. In either case, apparent clustering does not necessarily mean that the clusters are indeed different in a statistically significant manner. This is where statistical tests come into play to ensure that the clusters are indeed dissimilar, given the size of the data set and the variation of the variables. As ordination methods are applied to multivariate data, so must be the test, and matched to the type of data and cluster analysed. One of the most common multivariate tests (Warne et al., 2012) is the multivariate analysis of variance (MANOVA), a multivariate extrapolation of analysis of variance (ANOVA). Similarly to PCA, MANOVA uses the variance of the population, additionally, it assumes a multinormal distribution (Olson, 1974). Therefore, it is accepted to use this statistical test when clustering post PCA. Another problem with applying MANOVA is its internal assumption that the variables are independent (Finch, 2005), which is inherently problematic with closed set data, as discussed in chapter 13. Non-parametric variations of MANOVA (NPMANOVA) have been developed to mitigate some of these issues (Puri and Sen, 1971). The non-parametric analysis of similarities (ANOSIM) is a good fit to test groups inferred specifically from NMDS 
307 as both use ranking (Clarke, 1993; Buttigieg and Ramette, 2014). ANOSIM has essentially 308 only one assumption, that the ranges of ranked dissimilarities within groups are equal, or at 309 least very similar.

310 ANOSIM is an example of a permutation test, which overcomes various limitations of 311 parametric methods (Kowalewski and Novack-Gottshall, 2010; chapter 1.2 in Legendre and 312 Legendre, 2012). The permutation concept allows to compare sampled data against a 313 distribution drawn from random simulations rather than from a theoretical distribution, like the 314 Gaussian or the Poisson, and therefore to analyze data that does not satisfy the statistical assumptions underlying traditional parametric tests (Collingridge, 2013). It is also recommended to use a permutation test when sample sizes are small, or if the degrees of freedom are low. Since each data set can draw a distribution using permutations, we can use every possible statistical test on the alternative distribution, and thus create a permutation test. Therefore this concept can be used also in multivariate analysis. ANOSIM, for example, is a permutation test, as is PerMANOVA, the permutation alternative for the mentioned above MANOVA. ADONIS is suitable for mixed data seta as it relies on similarity indices, so the choice of a suitable similarity index makes it possible to compare variables which otherwise would not be comparable (Anderson, 2001).

\subsection{Transformations and standardization}

Variables used as input for ordination may need pre-treatment. The character of the pretreatment depends on the requirements of a particular method. Chapters 4.1.1 and 4.1.3, as well as Bialik et al. (2020), offer an example where the same data set needs different pretreatment for PCA and for DCA. Furthermore, some pre-treatment operations are specific to particular types of variables, such as in the case of a spectrogram (Abudulla et al., 2013) or ecological community composition. For the latter, pre-treatment considerations are explained in detail by Legendre and Gallagher (2001). Owing to the breadth of possibilities, we point to the most important situations requiring pre-treatment directly when explaining aspects of the respective ordination algorithm that necessitate it (see chapter 2.1 for PCA requirements). Furthermore, some pre-treatments, such as standardization to the same variance (see this chapter below), depend on the type of variable (explained in chapter 4.2), as variance cannot be meaningfully compared between variables measured on different scales. Pre-treatment of variables to fulfil the requirements of the chosen statistical method can be divided in two groups: transformation, which changes the shape of the distribution of a variable (e.g. from a log-normal to normal), and standardization, which does not change the shape of the distribution, but changes its position (Fig. 1). A common case of transformation is the 
normalizing transformation, or normalization (Legendre and Legendre, 2012), which is the adjustment of the variable's distribution to resemble the Gaussian (normal) distribution. In cases of positive skewness (long right tail) root transformations are useful, whereas in cases of negative skewness, log-transformations can be used. In addition to these general cases, there are many specific ones, e.g. bimodal distributions, where case-by-case choice may be necessary.

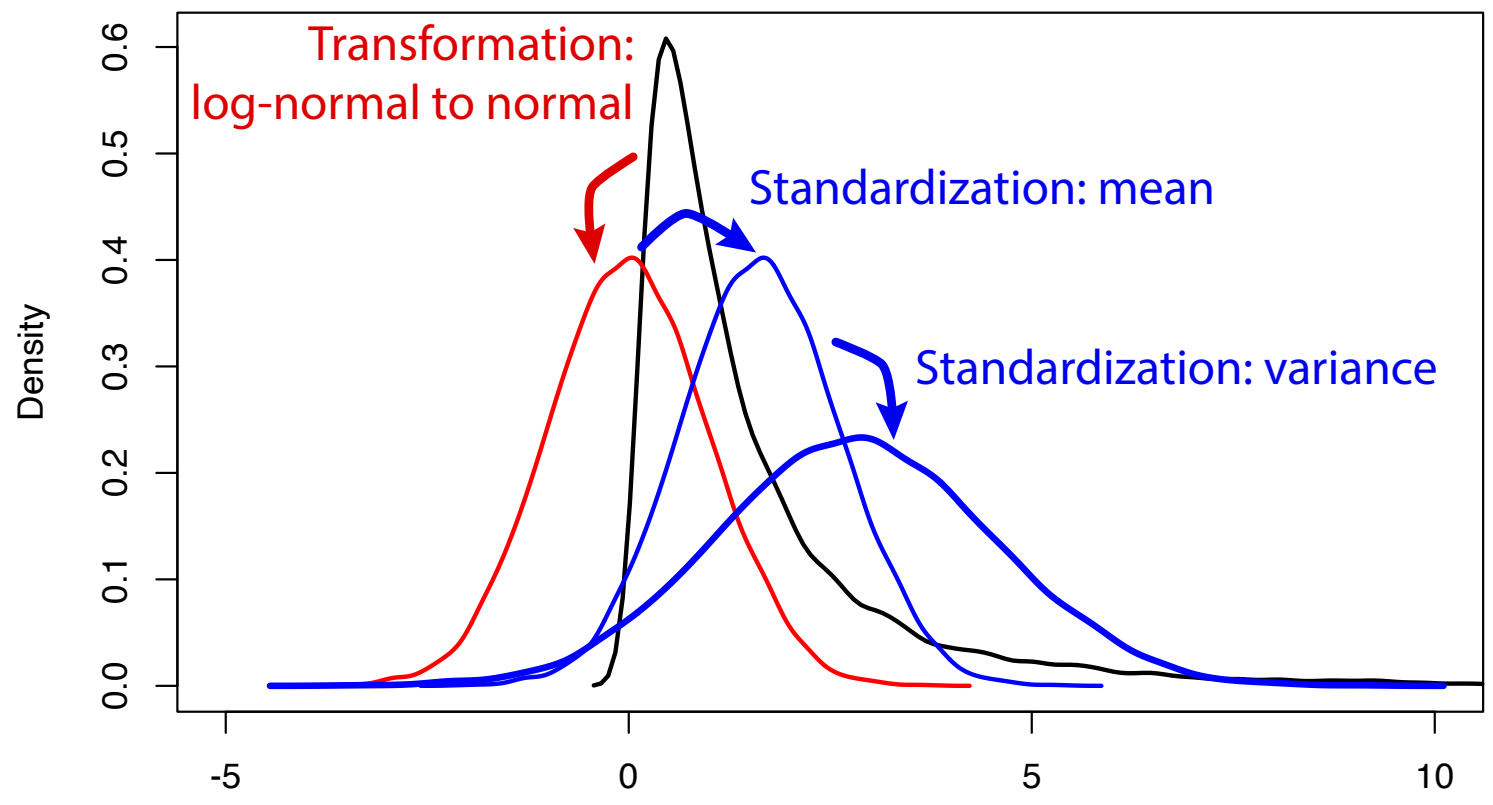

Fig. 1. Example of a common transformation from a log-normal distribution with mean equal 0 and standard deviation equal 1 to normal distribution. Log-transformation causes the mean and standard deviation of the generated normal distribution to be different from the original distribution, therefore two common standardization operations are shown: shifting (translation) of the distribution so that its mean equals zero and expanding it so that its standard deviation equals one. This combination allows the distribution to be used with methods requiring normality, while also preserving the original mean and variance.

Further examples of transformations include transformations aiming to achieve linear relationships between variables. For example, when the data set contains variables with uniform and exponential distributions, those with exponential distributions can be logtransformed. Ordination methods are typically robust with respect to shapes of distributions, with the notable exception of PCA (see above for details). If variables used as input for PCA have substantially different shapes of distributions, a correlation matrix instead of a covariance matrix should be used for calculations.

Normalizing transformation should not be confused with dividing elemental concentration by a reference, such as by PAAS (Australian Post-Archean Shale; Piper and Bau, 2013) for rare Earth elements, "conservative" elements, or TOC. This operation, confusingly, is also referred to as "normalization". The decision whether to apply this type of normalization is a matter of the research question and not a matter of fulfilling the requirements of a statistical method. 
369 Either original variable or variable modified using this type of normalization may be used in ordination analysis, but the incorporation of both at once should be avoided, as it will introduce spurious correlations. Spurious correlation may be also produced by division of one variable

372 by another one (Van der Weijden, 2002) and the need to perform it, as well as the effect it has

373 on the analysis should be considered individually, depending on the purpose of the analysis.

374 Standardization denotes any method of changing the absolute position of a variable's distribution without changing its shape. This may include translation, i.e. "shifting" so that the mean equals zero, or expansion/contraction (multiplying/dividing by a factor) so that the range of values falls into the $[-1,1]$ range. "Standardization" is sometimes taken to mean only a specific case of general standardization, that is standardizing to "z-scores", so that the variable's mean equals zero and its variance equals one. The choice of standardization method is dictated primarily by the statistical method and not by the type of variable, e.g. PCA based on a correlation matrix typically does not require any standardization, but some similarity indices used for NMDS may require standardization and then the choice of standardization method should follow the recommendations for the specific similarity index (see Jost et al., 2011 and Legendre and Legendre, 2012).

\subsection{Approach and methods}

\subsubsection{Data compilation}

In order to evaluate the use of ordination methods, sedimentological, geochemical and general geoscience journals were searched through using keywords "principal component analysis", "PCA", "non-metric multidimensional scaling", "NMDS", "detrended correspondence analysis", "DCA", and "ordination". Several queries were carried out using the Publish or Perish software v7 (Harzing, 2019) for each term in selected journals in the field, followed by manual review of individual manuscripts. We elected to use Google Scholar as the primary search venue due to its more inclusive definitions, the fact that it is not restricted by any pay wall and is a common resource used by many researchers. This resulted in partial export of the journal titles which had to be checked manually in several cases. The initial search yielded 5710 results from 23 
400 journals for all search terms. This was then cleaned to remove repetitions, yielding 4681

401 records (Tables S1 and S2 in Bialik, Jarochowska and Grossowicz, 2020). Numbers of articles

402 published per year in each journal were obtained from Scimago Journal and Country Rank

403 (https://www.scimagojr.com/). The time range was limited to focus only on the period of

404 proliferation of personal computing and introduction of widespread communication between

405 computers ("social computing", Sharma et al., 2016). From the narrower range of years, 117

406 were selected manually by the authors with no particular order (semi-randomly) from the list

407 generated by the initial search with supplementary searches to explore additional journals

408 from adjacent fields. In this manual phase, specific attention was given to newer studies

409 (published in 2020) to evaluate the current state of use. We have also tried to include not more

410 than one article from the same author in order to obtain a wider spectrum of methodologies

411 and minimize biases. Additionally, 100 manuscripts were selected fully randomly from the

412 initial query using a random number generator. Each manuscript was manually reviewed to

413 identify the methods used, workflow and software, type of data, what for and how the

414 ordination methods were used for, the number of data points, groups and variables for which

415 the analysis was carried out and how the data was presented and curated (Table S3, Bialik et

416 al., 2020). We included in our compilation only studies where PCA, NMDS or DCA were used,

417 all others were omitted. Primarily, our search focused on articles where fossils or sediment

418 were analyzed or that used methodologies that would also be relevant to the analysis thereof.

419 Manuscripts included in the final survey were limited only to those in which the methods were

420 implemented (i.e. not just mentioned and no review papers). We have not included studies

421 where ordination methods were part of a data processing workflow (as is sometimes the case

422 for X-ray absorption near edge structure (XANES), several paleomagnetic and image

423 processing methods among many others) and not the data analysis. In total 163 studies were

424 included in the final survey with 174 individual analyses listed collected from 43 different

425 journals in the fields for paleontology, paleoenvironment, sedimentology, and general geology.

426 Metadata for all the examined manuscripts included in the database is provided in the 427 associated data repository (Table S2, Bialik et al., 2020). Out of respect for our colleagues, 428 we anonymized the database and, in the following, we named only positive examples. Names 429 in DOls of the articles are listed separately and have been randomized.

\subsubsection{Data analysis}

431 All analyses have been performed using $R$ Software ( $R$ Core Team, 2020) and visualised 432 using the packages ggplot2 (Wickham, 2016) ang ggbiplot ( $\mathrm{Vu}, 2011)$. The $\mathrm{R}$ code and further 433 analyses and figures are available in a human-readable RMarkdown format as S4 in Bialik et 434 al. (2020). 


\subsection{Results - Findings from the survey of published ordination}

\section{7 analyses}

438 The number of publications utilizing ordination shows a consistent increase since the 439 beginning of the 1990s, this pattern is visible both in the analysis of the total number of 440 mentions and in the random selection sub-sample (Fig. 2).

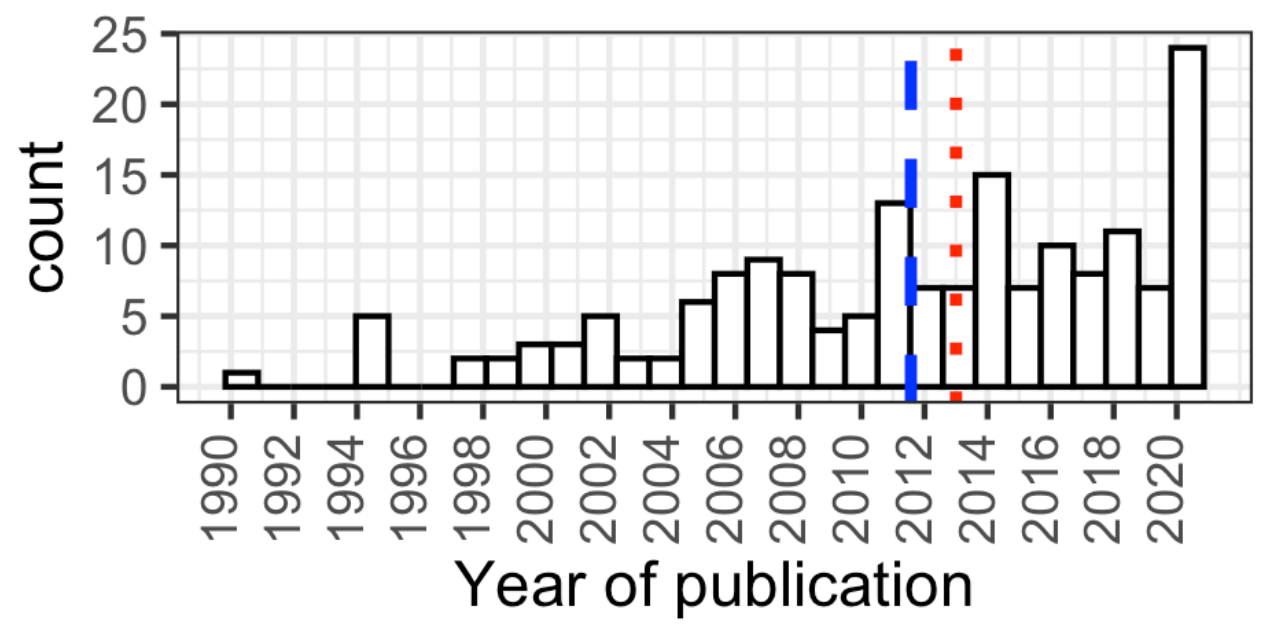

441

Fig. 2. Distribution of geoscience articles mentioning keywords used in our survey (see Methods for the list of keywords) through years $(n=174)$. The blue dashed line indicates the mean year (2011) and the red dotted line - the median (2013).

446 We identified three main uses for the ordination methods in the data set (Fig. 3): Clustering 447 and differentiation between groups (42\%), assessing the relation between variables (33\%) and generating indexes $(21 \%)$, in a few cases the authors set out to do more than one of these in the same study. Additionally, a smaller number of analysis set out to use ordination to identify most significant parameters $(n=5)$, test the relation between one parameter of interest to the other variables $(n=2)$ and assigning to pre-defined groups $(n=1)$. In the sampled studies, ordination was mostly commonly applied to elemental chemistry (37\%) and fossil assemblages $(31 \%)$, with other types of data including grain type $(8 \%)$, physical properties $(7 \%)$, mineralogy (4\%), organic molecules (3\%), isotopes (3\%) and others (Fig. 4 ). Some $18 \%$ of the analysis reviewed used more than one type of data. Most of the data sets $(65 \%)$ were compositional and another $17 \%$ were mixed. This wide swath of data types illustrates versatility and the power of ordination in geosciences and notably for work with sedimentary material. 


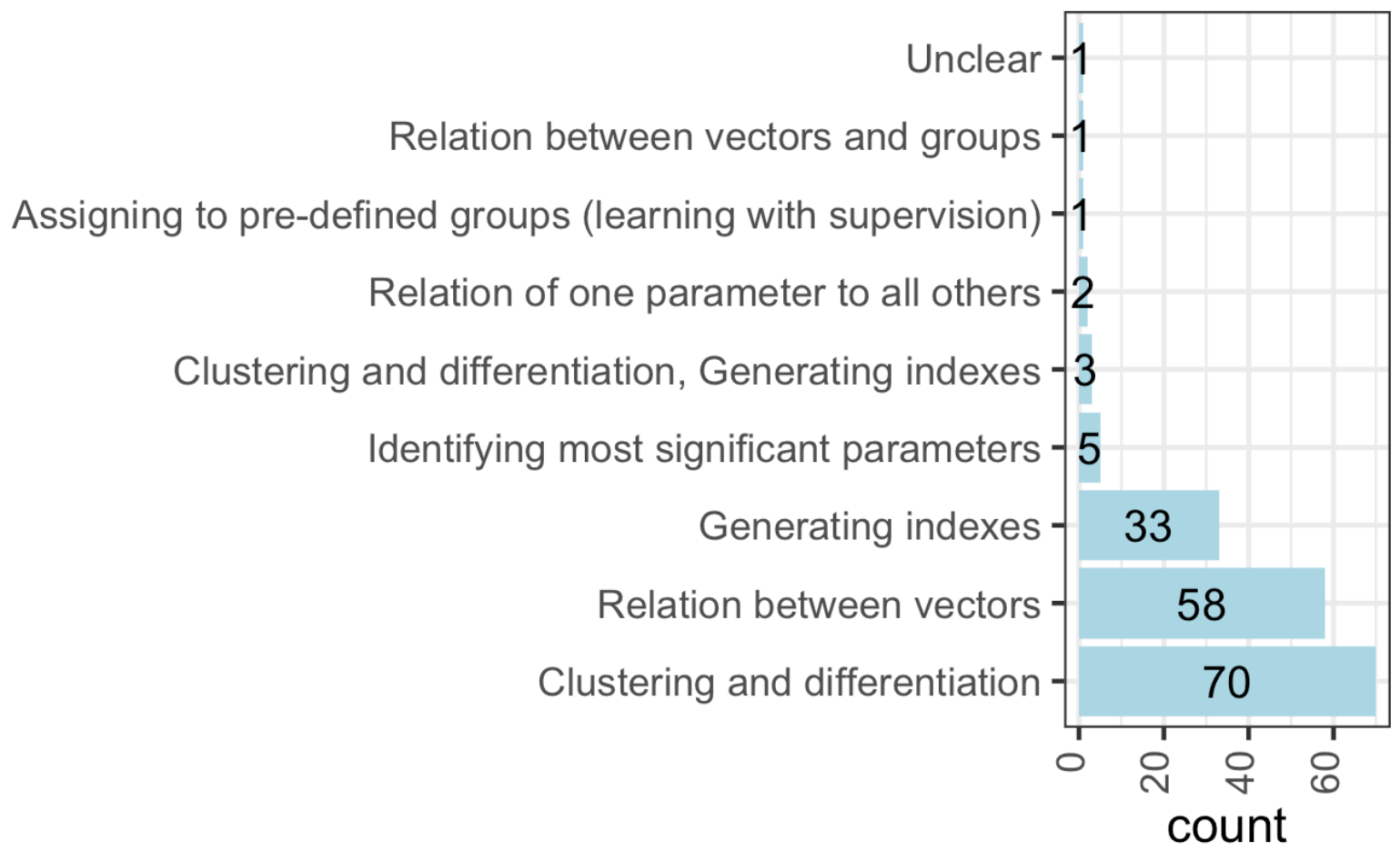

459

Fig. 3. Distribution of objectives for ordination analyses used in our survey $(n=174)$.

Of the three methods investigated (PCA, MNDS and DCA), the most ubiquitous is PCA, accounting for $84 \%$ of all results found in the initial search (Table S2, Bialik et al., 2020). The other two methods each account for around $12 \%$ of the references. In journals oriented towards palaeontology and paleoenvironment NMDS and DCA were encountered more often ( $16 \%$ and $22 \%$ of references, respectively) whereas in sedimentological, geochemical and general geoscience journals PCA exhibited the highest proportion (>90\%, Fig. 5). Interestingly, in palaeontology and paleoenvironment journals, DCA was encountered more often than NMDS, whereas in all other groups this method was encountered in fewer than $1 \%$ of all references, and completely absent from some journals. The average ( \pm standard deviation) number of articles per journal noting these ordination methods was $50 \pm 31$ for the sedimentological journals $(n=6), 270 \pm 212$ for palaeontology and paleoenvironment $(n=7)$, $262 \pm 124$ for geochemistry $(n=4)$, and $140 \pm 173$ for general geosciences $(n=6)$. Use of multiple ordination methods, an idea promoted in other disciplines (van Son and Halvorsen, 2014), was rarely observed in our database and searches, even when specifically queried. The use of multiple methods was mainly for analysis or ecological assemblages and applied to the same sample set (e.g., Zuschin et al., 2007; Abdelhady and Fürsich, 2014; Tyler and Kowalewski, 2014), with the common pairing being PCA and NMDS. One exception to this was Lanci et al. (2001) which used different methods for different data sets, although the reasoning was not explained in the text. Of 128 uses of PCA, only 5 included a test for normality. 


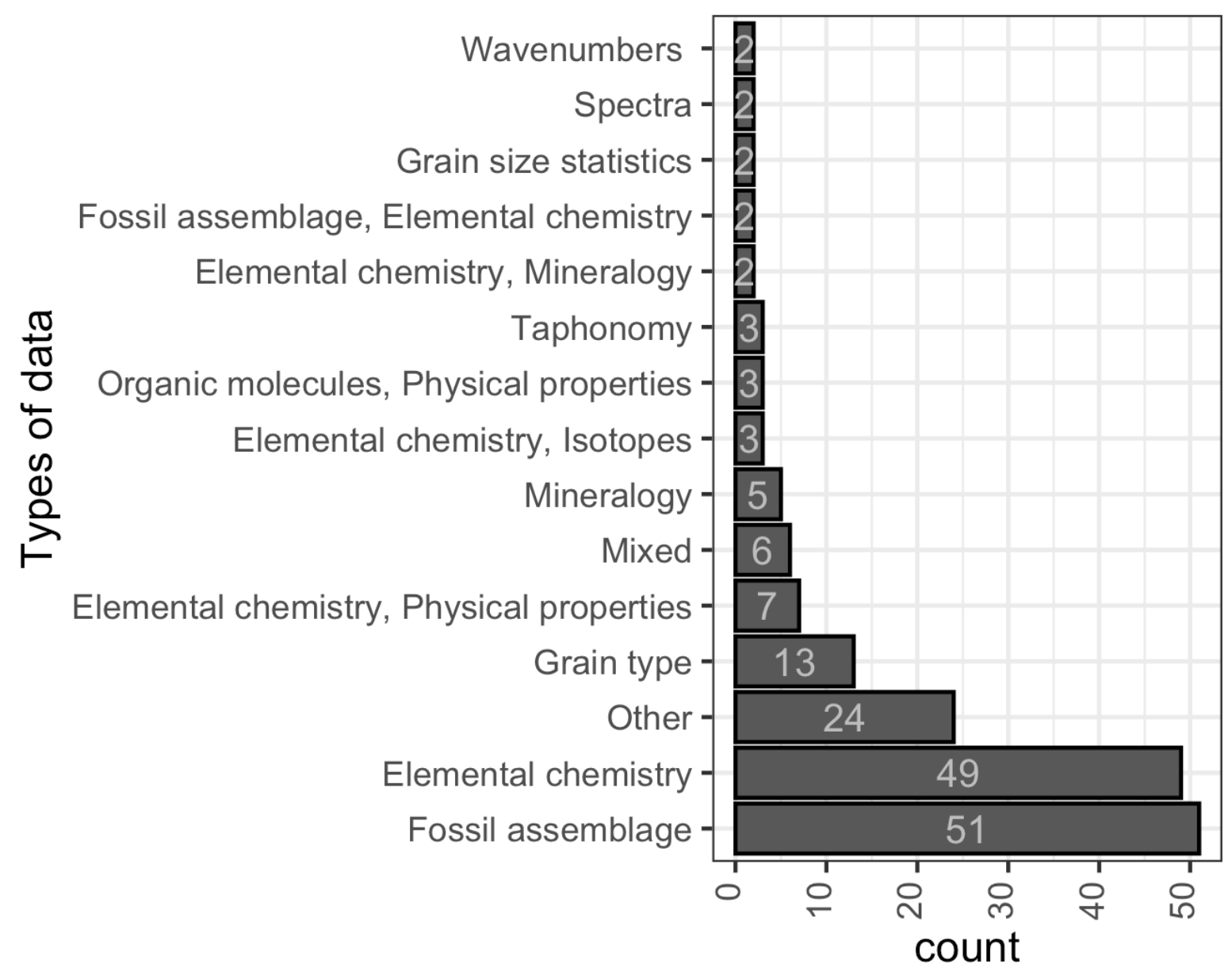

Fig. 4. Types of data in the survey $(n=174)$.

$484 \quad 3.2 .1$. Documentation of the analyses

485 Only $84 \%$ of all analyses provided complete information on the dimensions of the data set. 486 Among those, 24 analyses used data sets where the number of variables was larger than the 487 number of observations (Fig. 6). Among the 20 analyses using NMDS, 9 reported the similarity 488 index used, with Bray-Curtis being the most common. Lack of information about software used 489 for analysis was the most common case (38\%, Fig. 7), followed by PAST (14\%), R Software 490 (10\%). Among analyses which did report the software used, none of the 42 performed using 491 major coding-based packages, i.e. R, Matlab, SAS or SPSS, made the code available. 


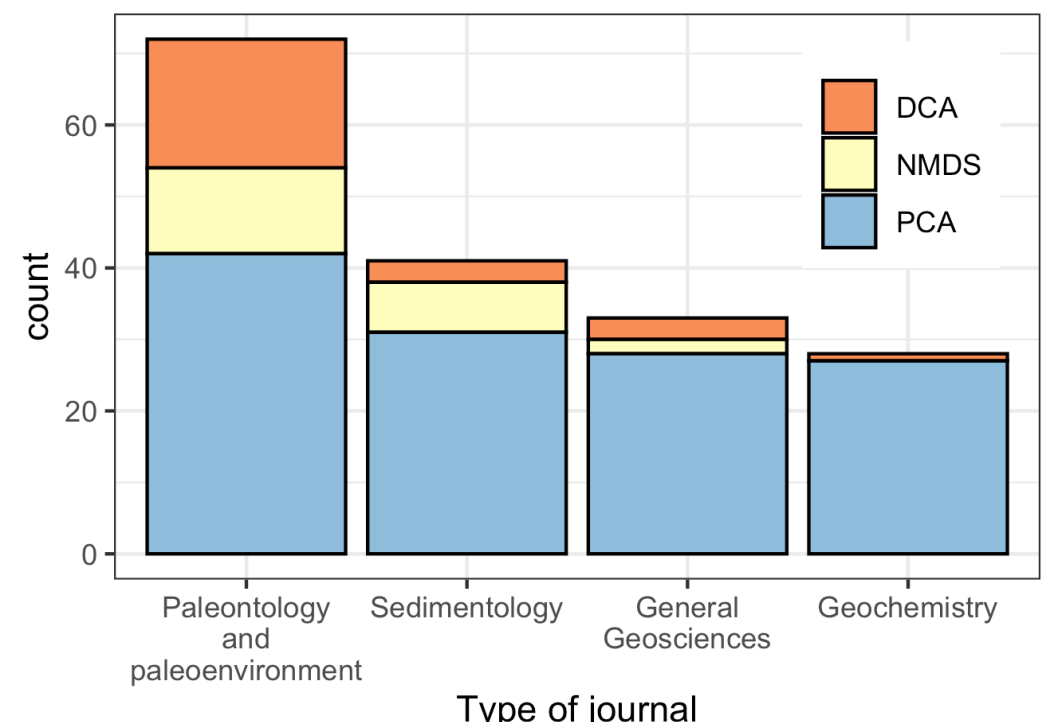

493 Fig. 5. Frequency of ordination types across journal disciplines $(n=174)$.

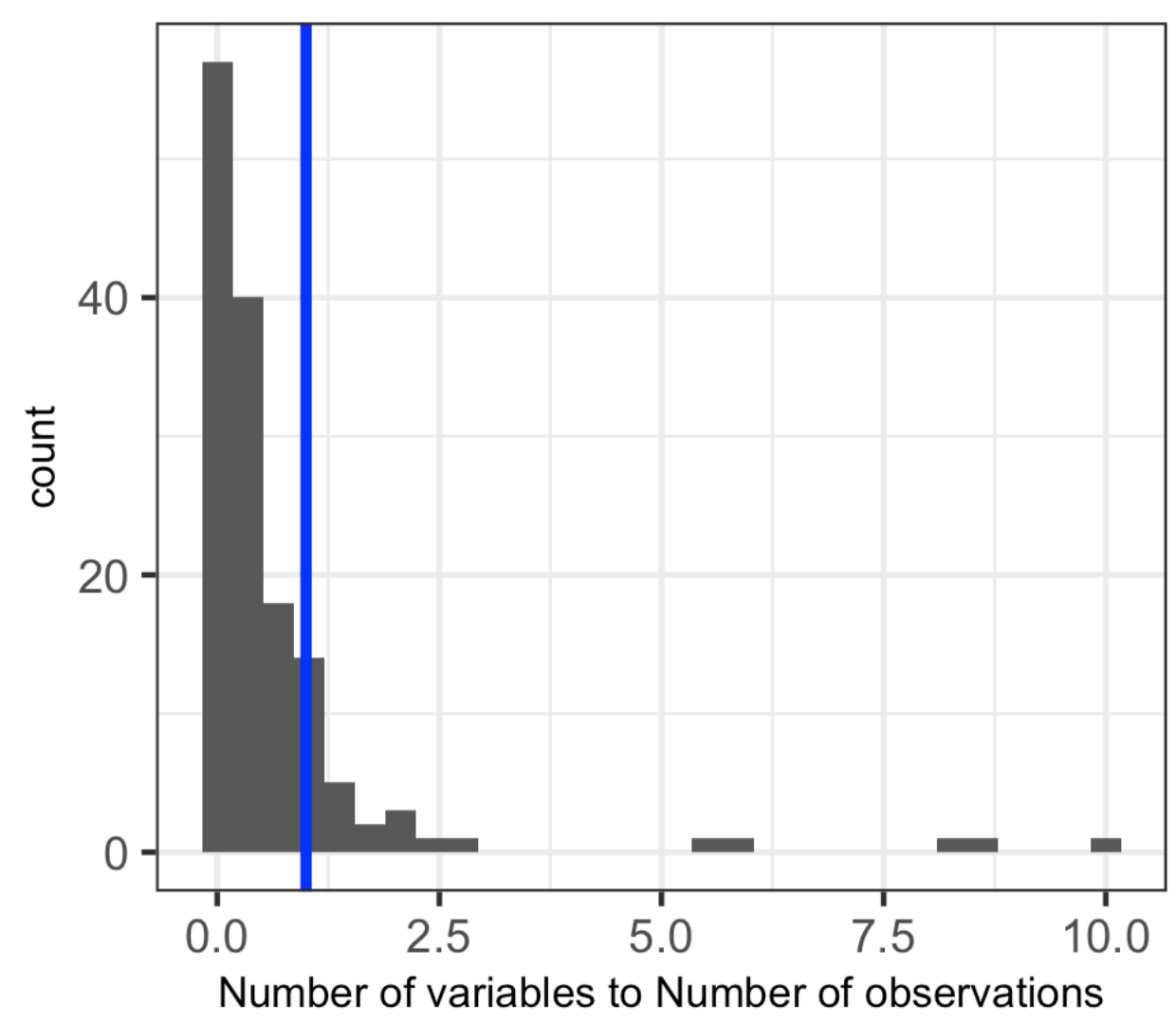

Fig. 6. Distribution of the ratio of the number of variables to the number of observations in analysed data sets $(n=146)$. The blue line indicates the threshold value of 1 .

\subsubsection{Data handling}

The majority (53\%) of surveyed studies did not provide the data used for analysis at all (Fig. 8). The second most common approach (35\%) was providing the data set directly in the article text or in the supplement, but in formats such as PDF or DOC, which are not readily imported into statistical software. The "golden standard" of placing a curated data set in an open access 
repository was followed only by $6(3 \%)$ of the analyses (Fig. 9). Throughout the entire time series, in each year, no data or data that is not readily processed (score 1) constituted more than a half of surveyed analyses. Only in the last two years (2019 and 2020) the average score rose above 1. Except for sedimentological journals $(49 \%, n=41)$, in all other categories articles with no data (score $=0$ ) formed the majority (Fig. 10). Articles with data in repositories were noted only in journals in general geosciences and in paleontology and paleoenvironment, but no significant differences could be detected in the data handling between journal types ( $p$ 509 $=0.64, \mathrm{n}=174$, Fisher's exact test).

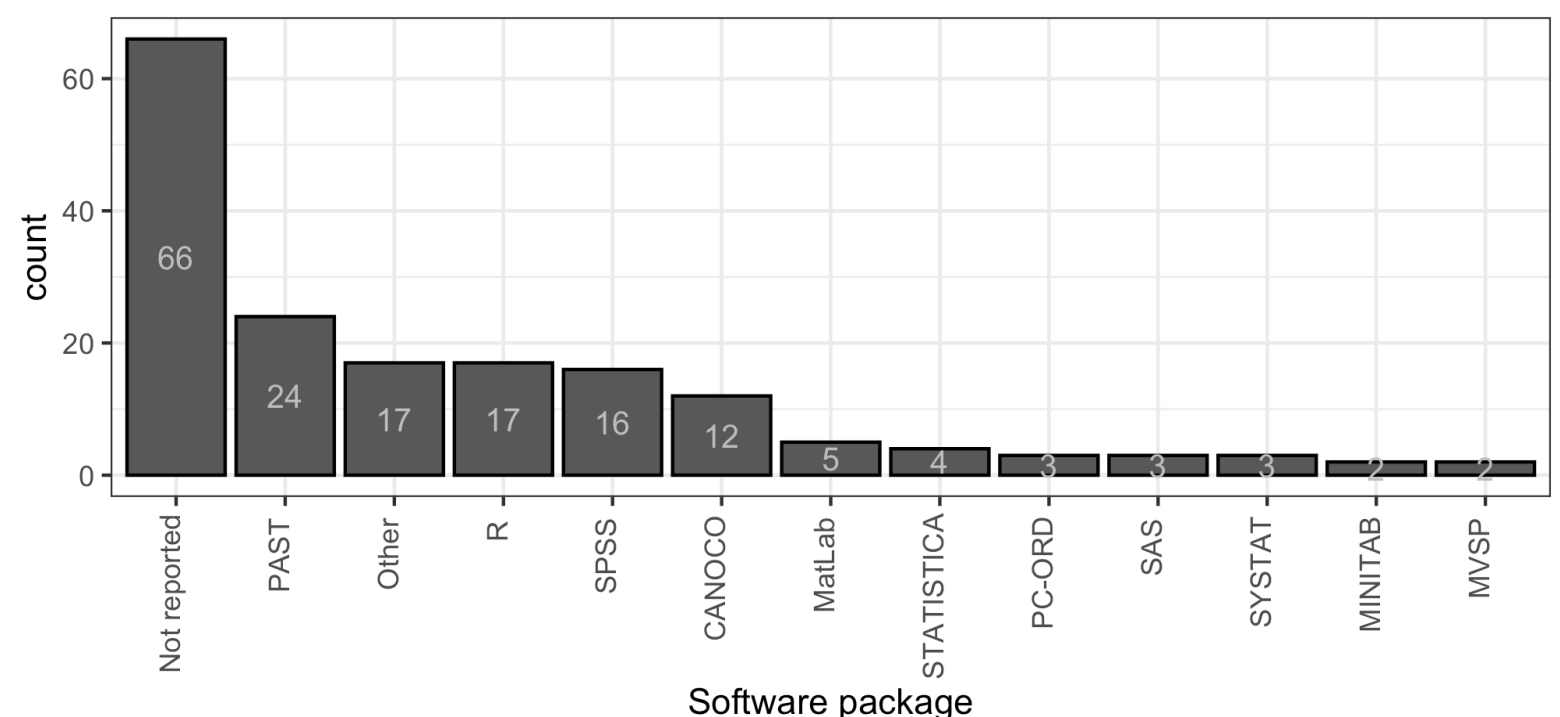

Fig. 7. Distribution of software packages reported by authors in the survey $(n=174)$.

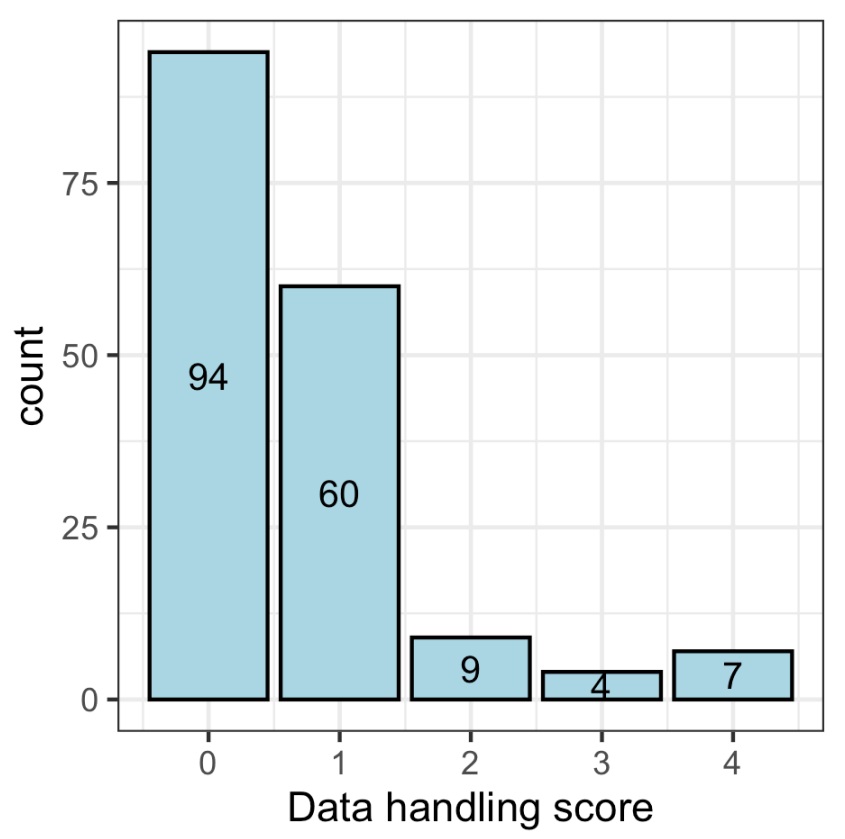

514 Fig. 8. Data handling score distribution ( $\mathrm{n}=174)$. 0 - no data; 1 - data in article text or in a non-machine 515 readable format in the supplement; 2 - machine-readable format in a supplement; 3 - 2, but no dead 516 cells; 4 - data curated in a repository. 


\subsubsection{Trends and patterns in the surveyed published ordination}

519 analyses

520 The use of ordination in the subdisciplines of Earth sciences investigated here is very diverse.

521 Despite reviewing only three types of ordination types, we still noted a fair variety of input, 522 application, methodology and objective.

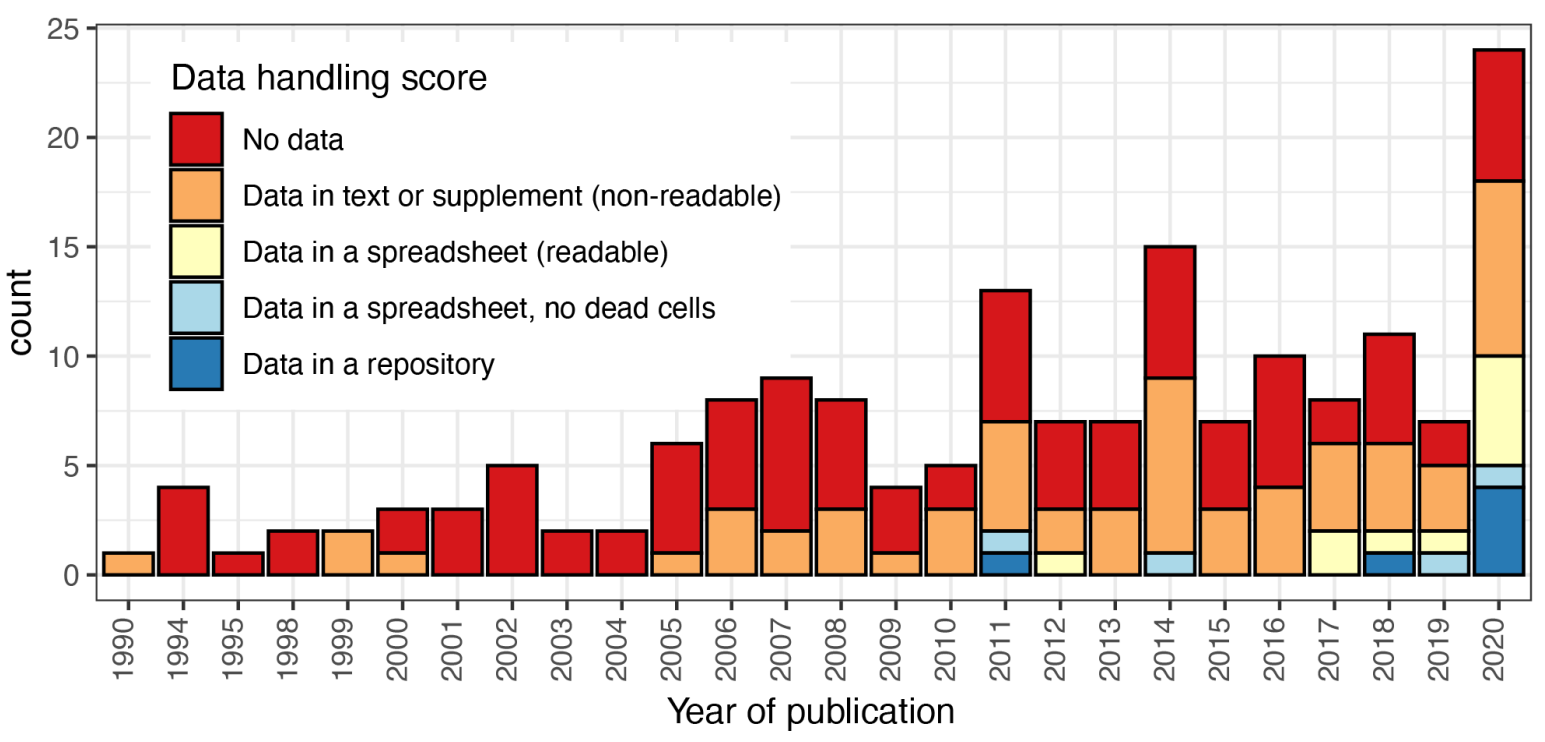

Fig. 9. Distribution of data handling scores across types of journals $(n=174)$. For legend, see Fig. 8.

There is a wide distribution of types of data. Most of the data types encountered are compositional, but non-compositional and mixed data types are not uncommon. It is expected that as more research will use a multi proxy approach, the fraction of the mixed data will further increase. This illustrates the need for diverse methodologies given the different nature of the

530 type of data used and transparency about the method application, as different data types 531 require different approaches. 
532

533

534

535

536

537

538

539

540

541

542

543

544

545

546

547

548

549

550

551

552

553

554

555

556

557

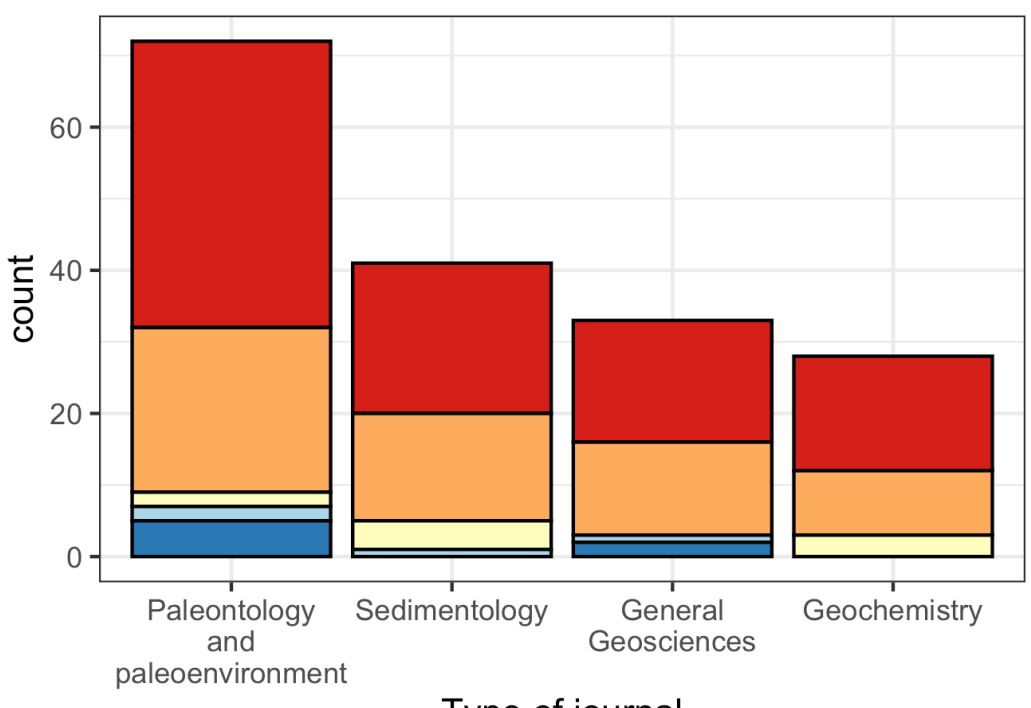

Type of journal

Fig. 10. Frequency of data handling scores across journal disciplines $(n=174)$. For legend, see Fig. 8.

PCA is not only the most commonly used method, it is also the one longest in use with the earliest reference from the late 1960s (Briggs, 1965; Read and Dean, 1968) whereas other methods were not encountered earlier than the late 1980s (Table S2, Bialik et al., 2020). While all three methods (or their precursors) were already discussed in the 1960s (Whittaker and Gauch, 1978), NMDS and DCA are more computationally demanding, which was a significant consideration at the time, leading to favouring PCA early on. This early adaptation probably had a significant role in cementing the popularity of PCA.

The higher numbers of references in which ordinations methods are mentioned over time could be the result of the increase in total number of publications, owing to increased access to publication and addition of new journals (Steen et al., 2013; Bornmann and Mutz, 2015). However, individually examined examples in our subset of journals analyzed do not seem to support this interpretation, as the increase is also observed at the single journal level, while its publication rate had not increased in a similar level. For example, the number of articles published per year by Palaeogeography, Palaeoclimatology, Palaeoecology had increased by $300 \%$ over the past twenty years (from 444 in 1999 to 1334 in 2019), where as the total number of references found in our survey in which PCA was mentioned had increased by an order of magnitude (from 3 in 2000 to 34 in 2020, Tab. 2). The Journal of Sedimentary Research has seen a decline in publication volume over the comparable period of around $40 \%$ but still exhibited an increase in mentions for PCA from between 0-1 per year in the late 1990s and early 2000 s to $0-5$ in the late 2010 s. These trends indicate an overall increase of awareness and use of ordination methods. Looking at the distribution of mentions per journal (Table S2, Bialik et al., 2020) in each field, and that the time span covered for all of these journals was rather similar ( $\sim 38$ years), even with the very large standard deviations it is clear there is still 
558 a lower inclination to use these methods in sedimentary research compared to other Earth 559 science fields.

560

\subsubsection{Common mistakes and issues}

\section{2}

563

564

565

566

567

568

569

570

571

572

573

574

575

576

577

578

579

580

581

582

583

584

585

586

587

588

589

590

591

\subsubsection{Basic metadata}

The most common problem we encountered in our review was poor documentation of the workflow used by the authors. Out of the 174 analyses evaluated (Table S2, Bialik et al., 2020), 66 do not include information on the software used. The most commonly used statistical programs were PAST (Hammer, Harper and Ryan, 2001), R (R Core Team, 2020), SPSS (IBM Corp., 2017) and CANOCO (ter Braak, 1989; ter Braak and Smilauer, 2012), in that order (Fig. 6). Matlab (Matlab, 2020) and Statistica (TIBCO Software, 2018) were also reported along inhouse, niche or non-statistical specific programs (e.g. ArcGIS). The preference for PAST and R Software, both freely available, as well as non-statistical software, such as Matlab (which many institutes acquire bulk licence for), suggests that accessibility plays an important role in which software is used. The bulk majority of authors provided no information of how they pretreated the data (if at all), how the analysis was carried out, or if the process was iterative or not, 16 did not even report the number of data points. This withholding of information made the evaluation of the validity of the ordination or its replicability impossible. Moreover, of the reviewed manuscripts that used code-based environments (such as Matlab or $\mathrm{R}$ ), none included their code in the supplement or provided it through a repository. This later part will change in the future as journals are adopting new transparency standards. Journals like Paleoceanography and Paleoclimatology adhere to the FAIR (Findability, Accessibility, Interoperability, and Reusability; sensu Wilkinson et al., 2016) principles with regard to data and require all data presented in the article to be available via a data repository. Other journals, like PeerJ, now also require the code to be included in the supplement or external repository. Another minor reporting problem we encountered was a mismatch between the reported use of ordination and their actual use. For example, the authors might write in the methods that they used the ordination method to group the results, but in actuality used it to examine the relations between variables.

In some cases where the data set was provided, it was not annotated or clearly marked - e.g. variables have mysterious names, the supplement is a folder of loose spreadsheets with cryptic names etc. We suggest to use data archives with established metadata structures, e.g. PANGAEA. Although data publication in PANGAEA is relatively new and none of the studies in our survey used it, it has several advantages over not-curated repositories such as Dryad: 
592

593

594

595

596

597

598

599

600

601

602

603

604

605

606

607

608

609

610

611

612

613

614

615

616

617

618

619

620

621

622

623

624

625

626

data curation assures that information is automatically exchanged with other databases, e.g. any biodiversity records are automatically transferred to Global Biodiversity Facility (GBIF) and the Ocean Biogeographic Information System (OBIS). This applies to many inventories, increasing the chances that the data set will be found and re-used. This is further facilitated by automatic registration of PANGAEA data sets in major scholarly databases and search engines, such as ORCID and Google Search.

Only a handful of the data sets in surveyed analyses were fully compliant with the FAIR data principles (Wilkinson et al., 2016), i.e. it was possible to find them through a database search, identify the data structure, and reuse them based on the metadata and the license. These positive examples included Watkinson and Hall (2019), Čejka et al. (2020), Grau Galofre et al. (2020) and four others. The use of a repository addresses several issues. It offers an external quality control on the arrangement of the data, it makes it discoverable and available to the rest of the scientific community, and it does not lock it behind a paywall. The need for quality control is illustrated by the fact that in some studies the data set does not match what is stated about the analysis. e.g. the analysis has been produced on a cleaned data set with dead cells, such as values below detection limit, removed, but only the raw, not cleaned data set is provided. Data sets provided in the PDF format directly in the article (e.g., Bialik et al., 2012; Jarochowska, 2012) or in the supplement are not strictly machine-readable, as exporting into an editable format typically introduces mistakes and requires extensive cleaning of formatting.

\subsubsection{Dimensions}

Another issue encountered with the misuse of ordination was the number of variables being larger than the number of data points. This was encountered in 25 analyses, in addition to another 26 in which either the number of data points or variables was unknown. Since there are only $n-1$ degrees of freedom for $n$ variables, the total number of variables should be no more than $n-1$ (Legendre and Legendre, 2012). Analysis of the impact of the ratio between the number of variables to the number of samples found the optimal ratio should be at least 2 , with some going up to 6 (Cattell, 1978; Kline, 1979). Most studies found the ratio of 3 samples per variable is optimal (Shaukat, Rao and Khan, 2016; Björklund, 2019), but $41 \%$ of the studies analysed here that used PCA had lower ratios. It is not uncommon that, in multiproxy studies, multiple analyses are carried out on the samples and, at times, this leads to the analyses producing a greater number of variables than there are samples available. This is especially true for studies where the number of samples is limited or applications where the pretreatment is especially long and complex, such as organic geochemistry. Analyses with more variables than samples, in some studies, could have been avoided, since the number of variables was 
627 increased by the authors by including the measurements and ratios between said 628 measurements as separate variables.

\section{3.3.2.3. PCA}

630 In our review, most of the issues found were with regard to use and application of PCA. As 631 stated above, normality, or at least symmetry of the distribution, is not needed for NMDS or 632 DCA, but it is important when using PCA. Yet very few studies $(n=5)$ clearly reported having 633 tested for normality of individual variables (e.g., Klubi et al., 2018; Allafta and Opp, 2020) or 634 multivariate normality of the data set (Abdulla et al., 2013). The issue is further complicated 635 when closed sum (compositional) data are involved. Here we observed that most 636 sedimentological and paleoenvironmental studies sampled had compositional or a mixture of 637 compositional and non-compositional data. These data sets have variables that are not 638 independent of each other, as they are part of a sum of a constant (Aitchison, 1982). Examples 639 of this (Table 2) would be percents, which describe a very large fraction of all sedimentological, 640 geochemical and paleoecological data sets. True central moments of compositional data are 641 not straightforward euclidean geometrical products, such as mean and standard deviations 642 (Filzmoser, Hron and Reimann, 2009; Tolosana-Delgado, 2012). These issues can be 643 addressed using transformations (e.g. Dunkley Jones et al., 2008; Auer et al., 2019; Caron et 644 al., 2020), but these were rarely implemented. Furthermore, in some data sets the distribution 645 of variables could not be made multinormal even if these transformations would be applied, 646 such as mixtures of closed and open data sets. Non-parametric approach would have been 647 preferable in those cases rather than those which would make assumptions about the 648 distribution of variables (here referred to as quasi-parametric). However, parametric or quasi649 parametric methods are those which are usually implemented. It should be noted that not all 650 geochemical or sedimentological data sets are necerally closed, raw XRF measurements, for 651 example, reported in counts per seconds, are not part of a sum of a constant and would not be compositional.

654 In regard to the use of NMDS, the most common issue was the absence of reporting on the 655 distance matrix and the methods by which it was generated. This is of particular importance 656 with respect to the data type analyzed as different distance indexes will define the stress value. 657 However, some distance methods, such as Gower, will be more informative with mixed data sets (van de Velden, lodice D'Enza and Markos, 2019). Another issue was the use of the NMDS axes as indices. This is not wrong per se, information about the separation between the data points is present in the NMDS output. Unlike PCA, where the axes represent scaled linear combinations of the variables, NMDS axes in default implementations are more 
662 qualitative and not measured on a ratio scale. However, some implementations allow scaling 663 of NMDS axes as "half-change", e.g. the function postMDS in the vegan package (Oksanen 664 et al., 2019) centers and scales the axes so that one unit means halving the similarity 665 (Jarochowska et al., 2017). To substantiate interpretation of axes, their scores can be tested 666 for correlation with variables (Tyler and Kowalewski, 2014). Moreover, the choice of an 667 implementation can significantly impact the replicability of the axial direction. The metaMDS 668 implementation in the vegan package for $\mathrm{R}$ Software, testing multiple starting configurations, 669 which can be made reproducible by initiating the random number generator by a fixed seed 670 (Oksanen et al., 2019) can mitigate these issues, but information about these steps is often 671 not specified in methods.

\section{$672 \quad 3.3 .2 .5$. Hypothesis testing}

673 Rather often, the output of the ordination is not substantiated or tested, notably when relations 674 between variables are concerned, but also when clustering. While some studies did combine 675 ordination with other Euclidean and non-Euclidean clustering methods, and then performed a 676 statistical test to validate that the resulting groups are significantly different (e.g. More et al., 677 2018; Gosling et al., 2019), others did not. Furthermore, some circular reasoning was 678 encountered with a priori groups preassigned to the data and then treating the ordination 679 clusters as validation. This last is fundamentally erroneous as ordination is a data exploration 680 tool set and not a tool to validate dissimilarity. An intermediate state is present with a priori 681 groups where the researchers seek to understand the relation of the groups they 682 predetermined to the $n$-dimensional variable space. For the research question in this situation, 683 it does not matter if the groups are dissimilar, just how they relate to each other in respect to 684 the variables. But the a priori assignment can result in groups having no relation to the 685 clustering observed in the ordination - which undermines this application.

\subsubsection{Graphical presentation}

A persistent issue in many publications reviewed was the presentation of the ordination. One common issue was not presenting the ordination itself and giving only a partial presentation of the resulting outcome. These cases often do not show any graphical presentation of the ordination output and auxiliary information such as the scree plot in PCA is not shown. This was very common in papers where ordination was used to generate indices, in these it was common for elements of the ordination not to be shown and only the index value to be presented. In other cases, only the loading was reported in a table or variables were shown in the ordination space without the data points. This sort of presentation limits the possibility to evaluate how the variables interact with the data set. The opposite issue was also 
697 (for PCA and DCA). Presentation of ancillary information, such as the scree plot for PCA or 698 Shepard plot for NMDS was rarely encountered in the main text or supplement. More often 699 the authors would only report the total variance (for PCA) for an axis, whereas these plots can 700 inform the reader of the level of importance of each component, including the ones not shown. 701 Although this is not as egregious when the explained variance on one axis is very high (>90\%). 702 Another issue observed with graphical presentation, encountered less but still present, 703 drawing of "blobs" around groups arbitrarily, according to authors' own preferences. This does 704 not offer a reproducible and objective evaluation of separation. Better options would have been 705 to draw convex hulls (Tomašových, 2004; Arreguín-Rodríguez and Alegret, 2016) if the groups 706 are known. When they are not known, clustering/machine learning without supervision for 707 unknown groups (e.g., Höltke et al., 2016; Bertolini et al., 2020) would be a preferred way of 708 identifying the groups.

709 We also encourage considering common color blindness in data presentation. An example of 710 how to maintain a consistent color scheme legible to most color-blind readers using 711 RColorBrewer (Neuwirth, 2014) is offered in the proposed workflow illustrated in S5 (Bialik et 712 al., 2020).

\section{Proposed workflow}

716 To illustrate a workflow fitting a typical sedimentological analysis, we have used a data set 717 from Bialik et al. (2018), available as S4 in Bialik et al. (2020). This data set included 718 geochemical and sedimentological information from a section carried out on Albian carbonate 719 sequence. A summary of analyses is presented below, but codes and full results of each step 720 are provided in Bialik et al. (2020). The initial data set consisted of 90 observations and 17 721 variables. Of these, 8 were compositional (concentrations given in ppm or percent, both of 722 elements and mineralogies), another 3 were ratios of compositional variables, 5 were 723 independent ratios considered non-compositional (isotopic data) and 2 were classifications 724 (lithology and texture). Additionally, the isotopic data also included error values. This data set 725 was selected for its complexity to illustrate a sort of worst case scenario. The test set is 726 composed of a mixture of open and closed sets and the variables are not normally distributed. 727 We performed the analysis in R Software (R Core Team, 2020), using routines which are also available in other popular softwares such as Matlab or PAST. 
729 Variables and observations with empty cells were identified using the package pheatmap 730 (Kolde, 2019). As default implementations of ordination analyses cannot handle empty cells, 731 we made the decision to exclude two variables with the highest number of empty cells: $\delta^{25} \mathrm{Mg}$ 732 (\% DSM3) and inorganic carbon (IC, \%Wt). This left us with a $61 \times 12$ matrix, which was used 733 for further analysis. Two variables were defined on a categorical scale (lithological description 734 and packing according to Embry and Klovan, 1971). They were excluded from ordination and 735 used as descriptors of samples.

\section{$736 \quad$ 4.1.1 PCA}

737 To fulfill the assumptions of PCA, tests for multivariate normality were conducted using the 738 MVN package (Korkmaz et al., 2014), including Mardia's, Henze-Zirkler and Royston's tests. 739 The normality of individual variables was additionally tested using the Shapiro-Wilk test. 740 Variables with extreme positive skewness were transformed as follows: Calcite (\%) and Ni/Co 741 using cubic root transformation; Sr (ppm), Zr (ppm) and Mn (ppm) - using square root 742 transformation. Transformation was chosen iteratively by measuring the resulting skewness, 743 as skewness, or deviance from symmetry, is the parameter of the distribution that is the most 744 confounding for PCA (Legendre and Legendre, 2012). PCA was performed using the princomp 745 function of $\mathrm{R}$ Software and the correlation matrix, because the variables in the data set were 746 mixed (i.e. measured in different units).

\subsubsection{NMDS}

748 As NMDS does not require normally or symmetrically distributed variables, we used the 749 original, un-transformed data set as input for the metaMDS function in the vegan package 750 (Oksanen et al., 2019). This implementation differs from the original NMDS algorithm in that it 751 tries to find a stable solution using several random starts and standardizes the scaling in the 752 result. The random number generator was seeded at a fixed number to assure that the same 753 results are obtained every time, otherwise each new run of the NMDS would result in a 754 different ordination or flipping of the axes.

DCA was performed on the data set after normalizing transformation (see PCA above). Additionally, the variables were standardized to the same range $[0,1]$, but without standardizing their variance. We used the decorana function of the vegan package (Oksanen et al., 2019). 


\subsubsection{Results of the case study}

761 For the initial data set, multivariate normality, as well as normality of individual variables, was rejected at $\alpha=0.05$. Transformations of the most skewed variables did not improve the multivariate distribution and the transformed data set still failed a test for multinormality, but univariate normality could not be rejected for the transformed $\mathrm{Zr}$ content (test statistic 0.9839 , $p=0.6044$ compared to 0.9048 and $p=0.0002$ prior to transformation, $n-=61)$. The transformations reduced the skewness of the univariate distributions, but the non-linear relationships between variables (S5 in Bialik et al., 2020) is a warning that PCA may not be suitable for this data set.

769 PCA ordination plot is shown in Fig. 11A. The same plot with observations labeled by the 770 Embry and Klovan (1971) classification, as well as the scree plot and a visualisation of 771 loadings, is available in S6 (Bialik, Jarochowska and Grossowicz, 2020). PC1 explained only $77231.7 \%$ of the total variance, its highest loadings were ${ }^{87} \mathrm{Sr} /{ }^{86} \mathrm{Sr}$ Initial Value (0.36) and \%Calcite 773 (loading 0.34$)$ and lowest $-\mathrm{Ce} / \mathrm{Ce}^{*}(-0.42)$ and $\mathrm{Zr}$ content $(-0.40)$. These pairs of variables 774 defined therefore the largest proportion of variance in the data set, with limestone samples 775 grouping at high values of PC1 and low values of PC1 corresponding to a mixture of dolomite, 776 marly dolomite and dolomitic marl samples. PC2 explained $19.1 \%$ of the total variance and 777 was most influenced by $\mathrm{Sr}$ content (loading 0.41 ) and $\mathrm{MgCO}_{3}$ content in dolomite (loading 778 0.51). 
A

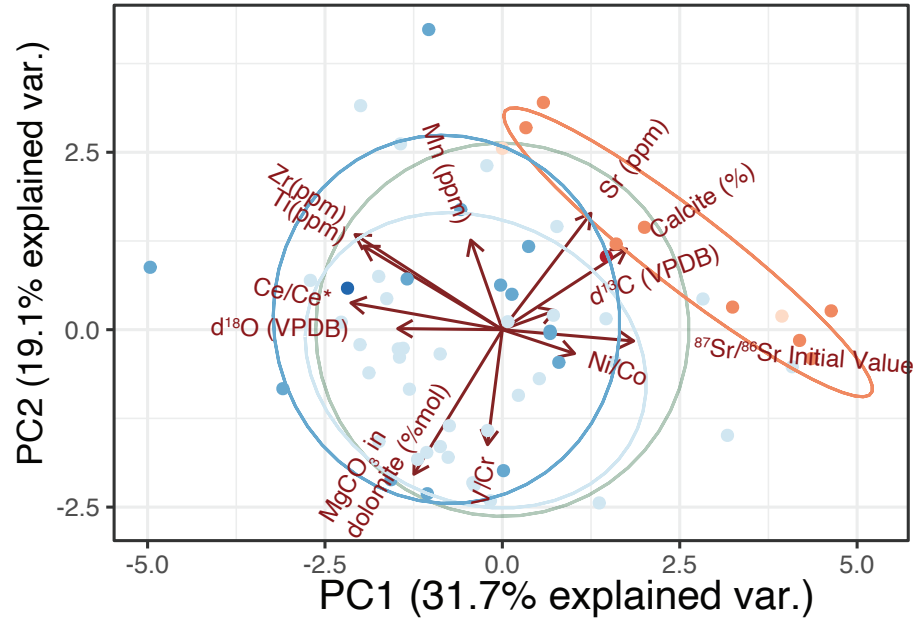

groups

$\rightarrow$ Marly limestone

$\rightarrow$ Limestone

- Dolomitic limestone

- Dolomite

- - Marly dolomite

- Dolomitic marl

B

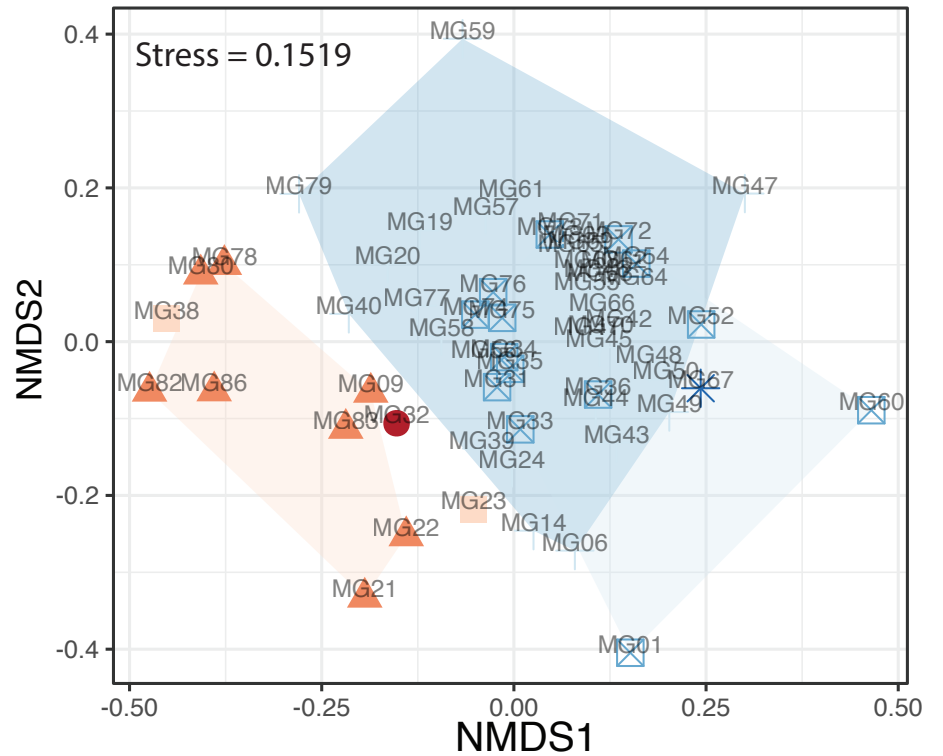

Lithology

Marly limestone

- Limestone

Dolomitic limestone

Dolomite

$\bigotimes$ Marly dolomite

* Dolomitic marl

C

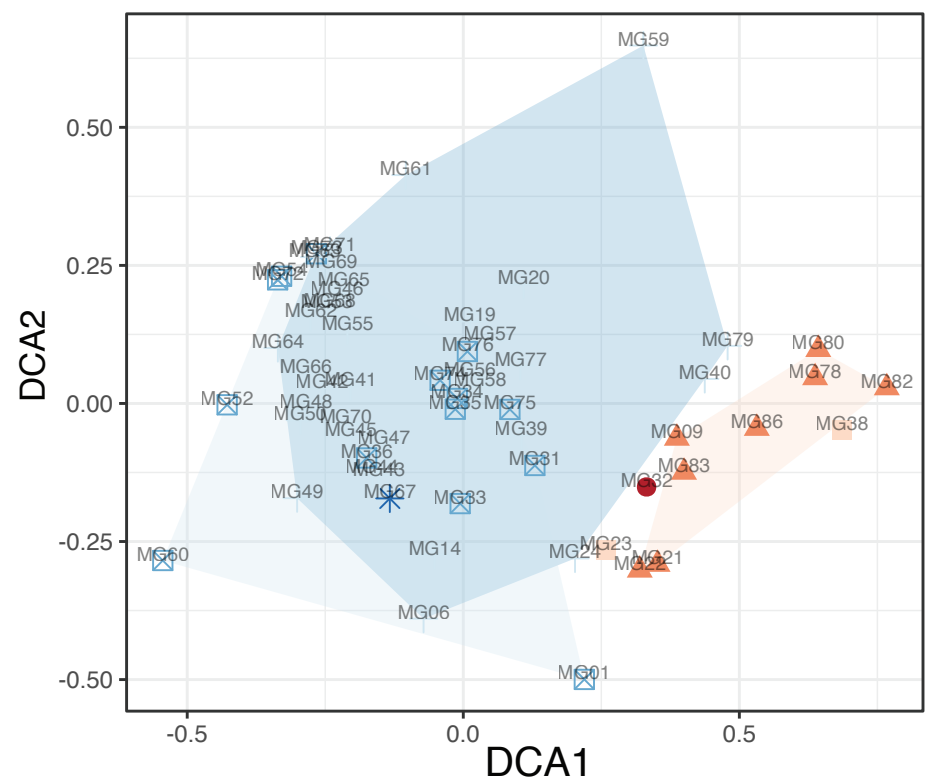

Fig. 11. Comparison of ordination results applied to the case study data set from Bialik et al. (2018), with observations grouped by lithology (61 observations, 12 variables). A. Principal Component Analysis with ellipses marking $68 \%$ confidence interval based on a multivariate t distribution. B. NonMetric Multidimensional Scaling with convex hulls. C. Detrended Correspondence Analysis with convex hulls. 
786 NMDS yielded an ordination with a stress value of 0.154 (Fig. 11B). Squared correlation 787 between fitted values and ordination distances was $R^{2}=0.905$, indicating a good representation of the distances between samples. Typically only observations are plotted in NMDS and DCA as these methods were designed to ordinate community matrices, but it is possible to obtain variable scores (Table S5 in Bialik et al., 2020). NMDS axis 1 corresponds to a gradient between limestone samples (high values of $\delta^{13} \mathrm{C}$ ) and marly dolostone samples (high $\delta^{18} \mathrm{O}$ values), with dolomite and marly limestone occupying intermediate positions along the gradient. High values of NMDS axis 2 corresponded to high $\mathrm{Ni} / \mathrm{Co}$ and $\mathrm{V} / \mathrm{Cr}$ content, represented by some dolomite samples. Low values of the NMDS axis 2 corresponded to high Mn content. DCA (Fig. 11C) resolved a similar gradient as NMDS, with limestone and marly dolostone defining the axis of the largest variance. Note that the gradient is flipped between Fig. 11B and Fig. $11 \mathrm{C}$, as the sign of the ordination axis has no meaning. In this analysis, DCA and NMDS cross-validate each other. It is an illustration of a case where PCA would not perform as well as NMDS or DCA, as initially identified by the non-linear relationships between variables and by the high skewness of individual variables.

\subsection{Consideration in compiling a multivariate data set - Types of} variables and how they are coded

Most introductory textbooks on multivariate data analysis implicitly assume variables are continuous, defined on a ratio scale and have no missing records or bounds. The best known parametric methods are also designed for such variables. But in geosciences deviations from these assumptions are plentiful.

808 Variables can be defined on four scales, sometimes called levels of measurement: nominal, 809 ordinal, interval and ratio variables. Nominal variables are categories which have no particular order. They are common in sedimentology and include for example rock types which do not represent any particular gradient, e.g. sedimentary, igneous, and metamorphic rocks. That is not to say there are no categories of rocks that have a natural order such as carbonate mudstone, wackestone and packstone according to the proportion of skeletal components.

814 Such variables are defined on an ordinal scale, but it is not possible to measure the distance 815 between them, e.g. we cannot say that packstone always has twice as many components as 816 wackestone. A mean or median calculated from a variable assigning samples to various categories in Dunham (1962) classification would be meaningless. 
818 Variables which have this property, i.e. values are equidistant, are termed interval. Their 819 values can be added and subtracted and means and medians calculated from them are 820 meaningful. But they do not have a true zero. The best known example is temperature 821 measured in ${ }^{\circ} \mathrm{C}$ or ${ }^{\circ} \mathrm{N}$, but in sedimentology perhaps the most common case are isotope ratios 822 such as $\delta^{13} \mathrm{C}$. If the variable can take minus values, it does not have a "true" zero. This is 823 important, e.g. for PCA, which cannot handle negative values, because a variable defined on 824 an interval scale can be standardized (here by moving, i.e. translating, the distribution to 825 positive values without changing its shape) to meet PCA requirements. Finally values which 826 can be added, subtracted, and have a "true" zero are called ratio variables and include 827 concentrations of elements or grain sizes.

828 Recognizing the scale at which a variable is measured is important to "code" it properly, i.e. 829 indicate the order and distance of categories in categorical variables. Coding refers here to 830 assigning numerical values to categories so that they can be processed by an ordination 831 method. For example, there are at least three different ways of coding grain size recorded in 832 the categories on the Wentworth (1922) scale: using phi $\left(\log _{2}\right.$ of the diameter) would make 833 them almost equi-distant (nearly interval), whereas assigning its middle value in metric units 834 to each category would produce a variable measured on an ordinal scale. This would produce 835 different distances between samples in NMDS, depending also on the similarity index used. 836 How the weights are assigned to variables may determine the conclusions of a study (Peng, 837 2015).

838 Some software packages allow defining the type of variable, which determines how it is 839 processed by the ordination method. For example, categorical variables in R Software are 840 stored as factors. These factors can be ordered, which corresponds to ordinal variables, or 841 not ordered, which corresponds to nominal variables. A common issue is that the type of 842 variable is not recognized correctly, e.g. because of a typo, and it can affect how the variable 843 is handled by ordination. It is recommendable to define types of variables explicitly, especially 844 when types are mixed within the data set (see workflow in 4.1). This allows avoiding problems 845 caused by visual spreadsheets, which may introduce errors by attempting to identify the types 846 of variables automatically (Ziemann et al., 2016). This automatic recognition is also a great 847 hindrance to reproducibility, since the same variable may be recognized as a different type 848 depending on the version and language settings of a particular computer.

849 A related problem is correct coding of zeros and missing values. The ordination analyses 850 described here, in their basic implementations, cannot handle missing values. There are tools allowing imputation of missing values. Some of them are specifically designed to assist the ordination algorithm (Stacklies et al., 2007; Filzmoser et al., 2018; Zhu et al., 2019), some may

853 be specifically designed to impute missing data of a given format. For example, software 854 packages for acquiring a diffraction signal will typically include imputation of single missing 
data points, and such specialized algorithms are likely to perform better than software for general use with any type of data. Compositional data in particular, dueto their properties, lend themselves to missing value imputation (Hron et al., 2010; Palarea-Albaladejo and MartínFernández, 2015). A very common error is to code missing values as zeros. Zeros are not "visible" in descriptive statistics such as the mean and standard deviation and therefore it often goes undetected that they do contribute to the distribution of a variable. A none-too-rare example would be geochemical analysis where concentrations are below the detection limit. Should these be coded as zeros, their distribution will artificially deviate from the normal distribution, which they might otherwise follow. If the software allows this, missing cells and zeros should be clearly distinguished.

Values below the detection limit are a common example of censored data, where a part of the distribution is not known. The sole coding of them (Often not numeric e.g. "<0.01", "LOD") is a common cause of errors in how the variable type is recognized. More importantly, it may be decisive for the outcome of the analysis whether these values are coded as a very small value, zeros or as absent. If very low values of a particular variable are an important characteristic of a set of samples, this information would be lost by replacing these values with empty cells. Furthermore, it would violate the assumptions of algorithms imputing missing values, as they assume that empty cells are randomly distributed. As values outside of detection limits are most common in compositional data, such cases are best treated with dedicated packages which allow handling them with less information loss (Templ et al., 2016; Filzmoser et al., 2018, and others cited below).

\subsection{Workflow recommendation}

A researcher interested in using ordination can use the following workflow (Fig. 12). The first stage of analysis of data should be identifying what kind of variables it contains (compositional, independent or mixed; nominal, ordinal, interval and ratio variables), as that will dictate many of the following steps. Selection of variables might be necessary as ideally the number of samples should be 3 times the number of variables (Shaukat, Rao and Khan, 2016), and under no circumstances equal or larger then the number of samples (Legendre and Legendre, 2012 and 3.3.2.2 here). Data sets which do not fulfil this criterion are sometimes referred to as "wide" and dedicated variations of PCA are available for them to allow exploratory analysis without a priori trimming the variables (Croux, Filzmoser and Fritz, 2013; Todorov and Filzmoser, 2013). If the data set contains nominal variables (e.g. facies names, most common minerals), they can either be ranked into an ordinal element or broken down to individual columns and turned into boolean (presence-absence) variables. If the former is employed, this will impact the data 
890 structure down-the-line and must be accounted for. Following that, the variables should be 891 examined individually to identify the shape of distributions (either graphically or by calculating 892 the skewness) and if any transformation might be needed. The variables can be plotted as 893 histograms, but in most earth science data sets, a spatial/temporal component exists. Plotting 894 the variables along these axes can give a first estimation of type of variability and inform later 895 interpretation of clusters. If the variables appear to have normal distributions, they should be 896 tested for multinormality. If they are clearly not normally distributed, examine the shape of the 897 distribution for pretreatment in the following stages.

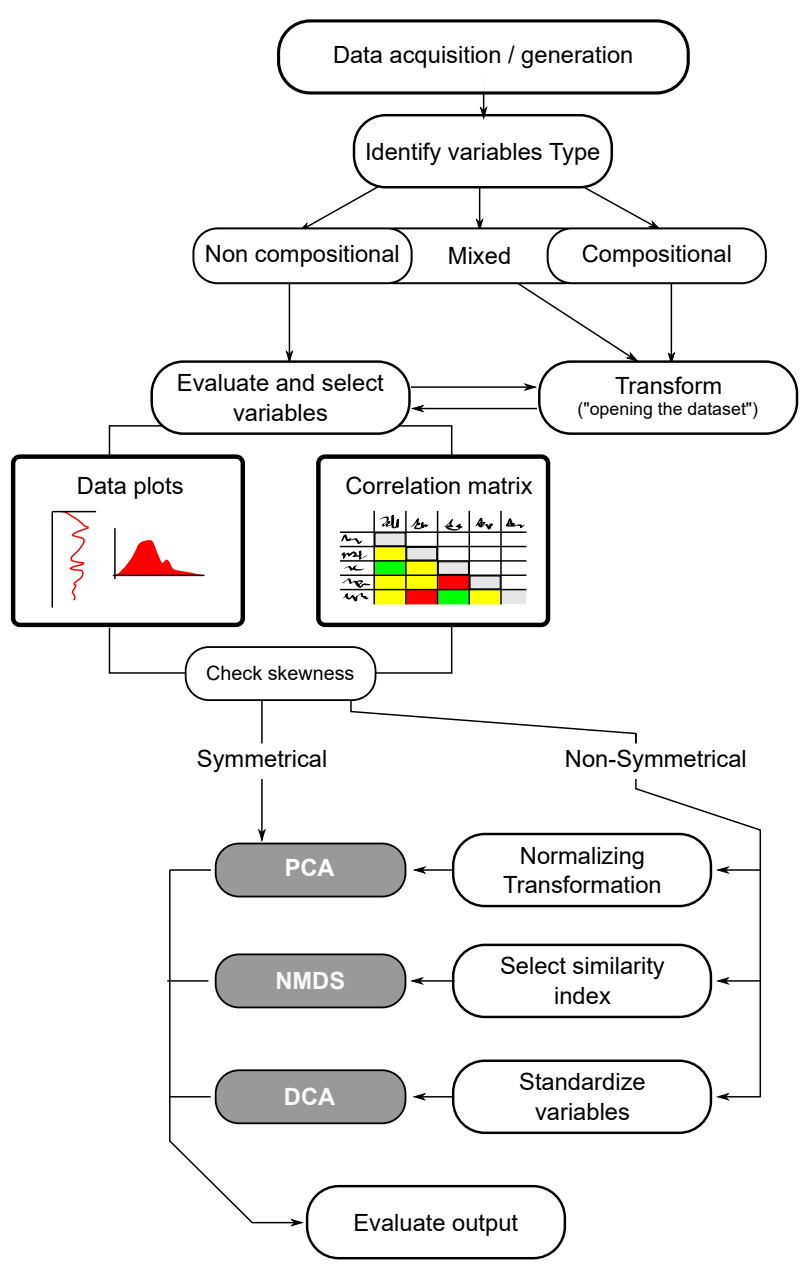

Fig. 12. Schematic workflow for the use of ordination in sedimentological, sedimentary geochemistry or paleoenvironmental research.

902 Next, generation of a correlation matrix is recommended. This can be done in any statistical software. Given the nonlinear nature of some sedimentological and geochemical data sets, and many of them being closed sets, a use of a ranked series correlation coefficient such as Spearman's or Kendall's coefficients than Pearson's for initial reconnaissance (Tolosana- 
906 Delgado, 2012) is recommended. Any pair of variables with high correlation coefficient should 907 be plotted to evaluate the correlation, $p$-values are not a reliable indicator with very large 908 (1000s or more data points) databases.

909 With an initial expectation of the outcome established, it is then possible to select the 910 ordination method and pretreat of the data. PCA relies on detecting linear relationships 911 between variables, since principal components are linear combinations of variables (Minchin, 912 1987). In contrast, NMDS and DCA perform better when relationships between variables are 913 not linear (such as redox-sensitive trace metals, which typically show logistic responses). As 914 this is not always known a priori, it is recommendable to compare the results of two or more 915 ordination methods (Patzkowsky and Holland, 2012), see also Abdelhady and Fürsich, 2014; 916 Tyler and Kowalewski, 2014). If the variables are measured on a ratio, interval or ordinal scale, 917 are non-compositional, and symmetrically (e.g. normally) distributed, they can be used for 918 PCA as is. Nevertheless, other ordination methods should also be used in addition. If not all 919 variables are symmetrically distributed, further processing is needed. Compositional or mixed 920 variables should be transformed, for example, using a root arcsin or an isometric log-ratio 921 transformation (Filzmoser, Hron and Reimann, 2009). After transformation, check the outcome 922 and distribution shape of the data by plotting the histograms or testing for normality. If the 923 result is suboptimal, attempt to use a different type of transformation. With the data optimized, 924 PCA could be performed. After performing PCA, examine the scree plot to see how much of 925 the explained variance is accounted for in each component to select the ones for evaluation. 926 If the distribution of variables cannot be adjusted sufficiently, or the eigenvalues are very low, 927 a method that does not require symmetrically distributed variables, such as NMDS or DCA, 928 should be employed. Simulations by Minchin (1987) and Patzkowsky and Holland (2012) 929 provided an empirical evaluation of the utility of different ordination methods for different types 930 of data sets. Patzkowsky and Holland (2012), for example, found that NMDS performed better 931 than DCA when two gradients (NMDS or DCA axes 1 and 2, respectively) represented similar 932 amounts of variation, whereas DCA performed better when one dominant gradient was 933 present. Empirical evaluations can be found in Bush and Brame (2010) and Tyler and 934 Kowalewski (2014), but clearly more are needed, especially for sedimentological data sets. 935 When generating the distance matrix for NMDS, see chapter 1.2 for a discussion of the choice 936 of similarity index. As a rule of thumb, if the data type is mixed, use Gower (van de Velden, 937 lodice D'Enza and Markos, 2019), if the data is boolean, use Jaccard, but consider how zeros 938 and missing values are coded, as they may influence the results (see chapter 4.2 here).

939 With the ordination performed, its results should be evaluated against the initial expectations 940 from the survey of the data. If they are different, first check if the output makes sense with the 941 data and if the initial expectations were wrong. Also consider if ordination output makes sense 942 from a geological perspective. In either case, it might be prudent to redo the analysis using a 
943 different transformation or with some of the variables excluded to evaluate if the results

944 replicate. If the desired output is clustering, perform the appropriate statistical test to make

945 sure the difference between the resulting groups is significant (see chapter 2.4).

946 With the ordination generated, it is important to report the detail of the workflow in the

947 publication. We strongly recommend to include the graphical representation of the ordination,

948 loading tables (for PCA and DCA), scree plot (PCA), etc. in the supplement if not the main text

949 of the manuscript. As advocates for open science, we also call upon authors, when possible,

950 to include the original data, preferably in a data repository.

\section{5. Concluding remarks}

952 Exploratory statistics and ordination in particular are of growing interest within the Earth 953 Science community. These methods offer an opportunity to analyze large multivariate data 954 sets, particularly with increased digitization of archival data and increasing data set sizes in 955 modern studies. The proliferation of freeware software and databases in Earth sciences and 956 rising interdisciplinarity in research creates an environment in which Earth scientists can 957 benefit from these tools. Students in these fields, in turn, could benefit from increased training 958 in multivariate statistics. Here, we presented a review of what ordiantion is, how it could be 959 used and what are some potential pitfalls in its application.

960 This work presents a survey of a large swath of studies using ordination in context of 961 sedimentology related studies. We found a diverse range of uses and applications. Although 962 most of the surveyed analyses used PCA, we find that NMDS and DCA are probably more 963 suitable for most geological data sets than PCA. We observed many cases of small mistakes 964 that could be avoided. Data reporting and access could benefit from new tools and policies 965 which would enhance reproducibility. Based on the finding from our review and survey we 966 propose a workflow (Fig. 11) for researchers new to ordination that are interested in unlocking 967 the potential in their sedimentological, sedimentary geochemistry or paleoenvironmental data. 968 For those who seek to deepen their understanding of the topics covered here, there are many 969 excellent reference books (Rencher, 2003; Hammer and Harper, 2007; Reimann et al., 2008; 970 Zar, 2010; Patzkowsky and Holland, 2012, 2012; Filzmoser, Hron and Templ, 2018; 971 Greenacre, 2018). All of these are useful introductory books written with geoscientists in mind 972 as well as discuss the use of accessible statistical software, notably PAST (Hammer, Harper 973 and Ryan, 2001) and R Software (R Core Team, 2020). 


\section{Acknowledgments}

975 This work had received no dedicated funding. OMB is supported by Marie Skłodowska Curie 976 Fellowship No. 101003394 (RhodoMalta). MG is supported by DFG Priority programme 977 (project DynaTrait, phase 2, 1704). EJ received funding from Deutsche 978 Forschungsgemeinschaft (project Ja 2718/3-1). The first two authors have made equal 979 contribution to this study.

\section{Data availability statement}

981 All the data sets, codes and RMarkdown documents are available as Bialik et al. (2020)

982 under https://doi.org/10.6084/m9.figshare.c.5250993.v1

983 RMarkdown documents can be previewed under https://rpubs.com/EmiliaJarochowska

984 Code for R Software can be also found in https://github.com/Bukoplot/ordination/ 


\section{References}

987 Abdelhady, A. A. and Fürsich, F. T. (2014) 'Macroinvertebrate palaeo-communities from the Jurassic succession 988 of Gebel Maghara (Sinai, Egypt)', Journal of African Earth Sciences, 97, pp. 173-193. doi: 989 10.1016/j.jafrearsci.2014.04.019.

990 Abdulla, H. A. N. et al. (2013) 'Transformations of the chemical compositions of high molecular weight DOM 991 along a salinity transect: Using two dimensional correlation spectroscopy and principal component analysis 992 approaches', Geochimica et Cosmochimica Acta, 118, pp. 231-246. doi: 10.1016/j.gca.2013.03.036.

993 Aitchison, J. (1982) 'The Statistical Analysis of Compositional Data', Journal of the Royal Statistical Society. Series 994 B (Methodological), 44(2), pp. 139-177.

995 Allafta, H. and Opp, C. (2020) 'Spatio-temporal variability and pollution sources identification of the surface sediments of Shatt Al-Arab River, Southern Iraq', Scientific Reports, 10(1), p. 6979. doi: 10.1038/s41598-02063893-w.

Alotaibi, K., Rayward-Smith, V. J. and de la Iglesia, B. (2011) 'Non-metric Multidimensional Scaling for PrivacyPreserving Data Clustering', in H., Y., W., W., and V., R.-S. (eds) Intelligent Data Engineering and Automated Learning. Berlin, Heidelberg: Springer. Lecture Notes in Computer Science, vol. 6936., pp. 287-298. doi: 1001 10.1007/978-3-642-23878-9_35.

1002 Amrhein, V., Trafimow, D. and Greenland, S. (2019) 'Inferential Statistics as Descriptive Statistics: There Is No Replication Crisis if We Don't Expect Replication', The American Statistician, 73(sup1), pp. 262-270. doi: 10.1080/00031305.2018.1543137.

1005 Anderson, M. J. (2001) 'A new method for non-parametric multivariate analysis of variance', Austral Ecology, 26(1), pp. 32-46. doi: 10.1111/j.1442-9993.2001.01070.pp.x.

Arreguín-Rodríguez, G. J. and Alegret, L. (2016) 'Deep-sea benthic foraminiferal turnover across early Eocene hyperthermal events at Northeast Atlantic DSDP Site 550', Palaeogeography, Palaeoclimatology, Palaeoecology, 451, pp. 62-72. doi: 10.1016/j.palaeo.2016.03.010.

1010 Auer, G. et al. (2019) 'Timing and Pacing of Indonesian Throughflow Restriction and Its Connection to Late 1011 Pliocene Climate Shifts', Paleoceanography and Paleoclimatology, 34(4), pp. 635-657. doi: 1012 10.1029/2018PA003512.

1013 Austin, M. P. (1968) 'An Ordination Study of a Chalk Grassland Community', The Journal of Ecology, 56(3), p. 739. 1014 doi: $10.2307 / 2258104$.

1015 Bertolini, G. et al. (2020) 'Controls on Early Cretaceous desert sediment provenance in south-west Gondwana, 1016 Botucatu Formation (Brazil and Uruguay)', Sedimentology. Edited by S. Dey, 67(5), pp. 2672-2690. doi: $101710.1111 /$ sed.12715.

1018 Bialik, O. M. et al. (2018) 'Mg isotope response to dolomitization in hinterland-attached carbonate platforms: 1019 Outlook of $\delta 26 \mathrm{Mg}$ as a tracer of basin restriction and seawater Mg/Ca ratio', Geochimica et Cosmochimica Acta, 1020 235, pp. 189-207. doi: 10.1016/j.gca.2018.05.024.

1021 Bialik, O. M., Jarochowska, E. and Grossowicz, M. (2020) 'Supporting data for: On the application and utility of 1022 ordination methods in sedimentology, paleontology and geochemistry', Figshare. doi: 1023 10.6084/m9.figshare.c.5250993.

1024 Bialik, O. M., Korngreen, D. and Benjamini, C. (2012) 'Lithofacies and cyclicity of Mohilla evaporite basins on the rifted margin of the Levant in the Late Triassic, Makhtesh Ramon, southern Israel', Sedimentology, 59(7), pp. 2097-2124. doi: 10.1111/j.1365-3091.2012.01336.x.

Birks, H. J. B. (1985) 'Recent and possible future mathematical developments in quantitative palaeoecology', Palaeogeography, Palaeoclimatology, Palaeoecology, 50(1), pp. 107-147. doi: 10.1016/S0031-0182(85)800080 .

1030 Bitušík, P. et al. (2018) 'Tracking human impact in a mining landscape using lake sediments: A multi-proxy 
1033 Björklund, M. (2019) 'Be careful with your principal components', Evolution, 73(10), pp. 2151-2158. doi: 1034 10.1111/evo.13835.

1035 ter Braak, C. J. F. (1986) 'Canonical Correspondence Analysis: A New Eigenvector Technique for Multivariate Direct Gradient Analysis', Ecology, 67(5), pp. 1167-1179. doi: 10.2307/1938672.

1037 ter Braak, C. J. F. (1989) 'CANOCO - an extension of DECORANA to analyze species-environment relationships', 1038 Hydrobiologia, 184(3), pp. 169-170. doi: 10.1007/BF02392953.

1039 ter Braak, C. J. F. and Prentice, I. C. (1988) 'A Theory of Gradient Analysis', advances in ecological research, 18, pp. 271-317. doi: 10.1016/S0065-2504(08)60183-X.

1041 ter Braak, C. J. F. and Smilauer, P. (2012) Canoco reference manual and user's guide: software for ordination, 1042 version 5.0. Microcomputer Power.

1043 Bray, J. R. and Curtis, J. T. (1957) 'An Ordination of the Upland Forest Communities of Southern Wisconsin', 1044 Ecological Monographs, 27(4), pp. 325-349. doi: 10.2307/1942268.

1045 Briggs, L. I. (1965) 'Heavy Mineral Correlations and Provenances', Journal of Sedimentary Research, 35(4), pp. 1046 939-955. doi: 10.1306/74D713B6-2B21-11D7-8648000102C1865D.

1047 Brown, C. E. (1998) Applied Multivariate Statistics in Geohydrology and Related Sciences. Berlin, Heidelberg: Springer Berlin Heidelberg. doi: 10.1007/978-3-642-80328-4.

1049 Bush, A. M. and Brame, R. I. (2010) 'Multiple paleoecological controls on the composition of marine fossil assemblages from the Frasnian (Late Devonian) of Virginia, with a comparison of ordination methods', Paleobiology, 36(4), pp. 573-591. doi: 10.1666/07022.1.

Buttigieg, P. L. and Ramette, A. (2014) 'A guide to statistical analysis in microbial ecology: a community-focused, living review of multivariate data analyses', FEMS Microbiology Ecology, 90(3), pp. 543-550. doi: 10.1111/15746941.12437.

Caron, M. et al. (2020) 'Quantifying Provenance and Transport Pathways of Holocene Sediments From the Northwestern Greenland Margin', Paleoceanography and Paleoclimatology, 35(5). doi: 10.1029/2019PA003809.

Cattell, R. B. (1978) The Scientific Use of Factor Analysis in Behavioral and Life Sciences. Boston, MA: Springer. doi: 10.1007/978-1-4684-2262-7.

Chao, A. et al. (2006) 'Abundance-Based Similarity Indices and Their Estimation When There Are Unseen Species in Samples', Biometrics, 62(2), pp. 361-371. doi: 10.1111/j.1541-0420.2005.00489.x.

1061 Chatfield, C. (1995) Problem Solving: A Statistician's Guide. Chapman and Hall.

1062 Clarke, K. R. (1993) 'Non-parametric multivariate analyses of changes in community structure', Austral Ecology, 18(1), pp. 117-143. doi: 10.1111/j.1442-9993.1993.tb00438.x.

1064 Collingridge, D. S. (2013) 'A Primer on Quantitized Data Analysis and Permutation Testing', Journal of Mixed 1065 Methods Research, 7(1), pp. 81-97. doi: 10.1177/1558689812454457.

1066 Cooper, M. M. (2018) 'The Replication Crisis and Chemistry Education Research', Journal of Chemical Education, 95(1), pp. 1-2. doi: 10.1021/acs.jchemed.7b00907. pp. 202-214. doi: 10.1080/00401706.2012.727746.

Dexter, E., Rollwagen-Bollens, G. and Bollens, S. M. (2018) 'The trouble with stress: A flexible method for the evaluation of nonmetric multidimensional scaling', Limnology and Oceanography: Methods, 16(7), pp. 434-443. doi: 10.1002/lom3.10257.

1073 Duneman, G. H. (1989) Principal component analysis, Journal of Classification. Sage Publications - quantitative 1074 applications in the social-sciences no. 69 (1).

1075 Dunham, R. J. (1962) 'Classification of Carbonate Rocks According to Depositional Textures', in Ham, W. E. (ed.) 1076 Classification of Carbonate Rocks - A Symposium. AAPG Memoir 1, pp. 108-121.

1077 Dunkley Jones, T. et al. (2008) 'Major shifts in calcareous phytoplankton assemblages through the Eocene1078 Oligocene transition of Tanzania and their implications for low-latitude primary production', Paleoceanography, 
23(4), p. n/a-n/a. doi: 10.1029/2008PA001640.

Egozcue, J. J. (2009) 'Reply to "On the Harker Variation Diagrams; ..." by J.A. Cortés', Mathematical Geosciences, 41(7), pp. 829-834. doi: 10.1007/s11004-009-9238-0.

Field, J. G., Clarke, K. R. and Warwick, R. M. (1982) 'A Practical Strategy for Analysing Multispecies Distribution Patterns', Marine Ecology Progress Series, 8(1), pp. 37-52.

Filzmoser, P., Hron, K. and Reimann, C. (2009) 'Univariate statistical analysis of environmental (compositional) data: Problems and possibilities', Science of the Total Environment, 407(23), pp. 6100-6108. doi: 10.1016/j.scitotenv.2009.08.008.

Filzmoser, P., Hron, K. and Templ, M. (2018) Applied Compositional Data Analysis. Cham: Springer International Publishing (Springer Series in Statistics). doi: 10.1007/978-3-319-96422-5.

Finch, H. (2005) 'Comparison of the Performance of Nonparametric and Parametric MANOVA Test Statistics when Assumptions Are Violated', Methodology, 1(1), pp. 27-38. doi: 10.1027/1614-1881.1.1.27.

Gauch, H. G. (1982) Multivariate Analysis in Community Ecology. Cambridge, UK: Cambridge University Press.

Gauch, H. G. and Whittaker, R. H. (1972) 'Comparison of Ordination Techniques', Ecology, 53(5), pp. 868-875. doi: $10.2307 / 1934302$.

Gosling, W. D., Cornelissen, H. L. and McMichael, C. N. H. (2019) 'Reconstructing past fire temperatures from ancient charcoal material', Palaeogeography, Palaeoclimatology, Palaeoecology, 520, pp. 128-137. doi: 10.1016/j.palaeo.2019.01.029.

Gower, J. C. (1987) 'Introduction to Ordination Techniques', in Legendre P., L. L. (ed.) Develoments in Numerical Ecology. Berlin, Heidelberg: NATO ASI Series (Series G: Ecological Sciences) 14. Springer, pp. 3-64. doi: 10.1007/978-3-642-70880-0_1.

Greenacre, M. (2018) Compositional Data Analysis in Practice. Chapman and Hall, CRC Interdisciplinary Statistics. Griffiths, J. C. (1947) 'Sedimentary petrography and the oil industry', Journal of Sedimentary Research, 17(1), pp. 29-34. doi: 10.1306/D4269297-2B26-11D7-8648000102C1865D.

Gupta, A. K. and Thomas, E. (1999) 'Latest Miocene-Pleistocene Productivity and Deep-Sea Ventilation in the Northwestern Indian Ocean (Deep Sea Drilling Project Site 219)', Paleoceanography, 14(1), pp. 62-73. doi: 10.1029/1998PA900006.

Hammer, $\varnothing$. and Harper, D. A. T. (2007) Paleontological Data Analysis, Paleontological Data Analysis. Blackwell Publishing Ltd. doi: 10.1002/9780470750711.

Hammer, Ø., Harper, D. A. T. and Ryan, P. D. (2001) 'PAST: paleontological statistics software package for education and data analysis', Palaeontologia Electronica, 4(1). Available at: http://palaeoelectronica.org/2001_1/past/issue1_01.htm.

Harzing, A. W. (2019) Publish or perish. Available at: https://harzing.com/resources/publish-or-perish.

Hastie, T., Tibshirani, R. and Friedman, J. (2009) The Elements of Statistical Learning: Data Mining, Inference, and Prediction. New York, NY: Springer Series in Statistics (Springer Series in Statistics). doi: 10.1007/978-0-38784858-7.

Hill, M. O. (1979) DECORANA - A FORTRAN program for detrended correspondence analysis an reciprocal averaging. Section of Ecology and Systematics, Cornell University.

Höltke, O., Salvador, R. B. and Rasser, M. W. (2016) 'Paleobiogeography of Early/Middle Miocene terrestrial gastropods in Central Europe: An approach using similarity indices', Palaeogeography, Palaeoclimatology, Palaeoecology, 461, pp. 224-236. doi: 10.1016/j.palaeo.2016.08.027.

Hotelling, H. (1933) 'Analysis of a complex of statistical variables into principal components.', Journal of Educational Psychology, 24(6), pp. 417-441. doi: 10.1037/h0071325.

Ibanez, F. (1971) 'Effet des transformations des données dans l'analyse factorielle en écologie planctonique', Cah. Oceanogr, 23, pp. 545-561.

IBM Corp. (2017) IBM SPSS Statistics for Windows. Armonk, NY: IBM Corp. Available at: 
1125

1126

1127

1128

1129

1130

1131

1132

1133

1134

1135

1136

1137

1138

1139

1140

1141

1142

1143

1144

1145

1146

1147

1148

1149

1150

1151

1152

1153

1154

1155

1156

1157

1158

1159

1160

1161

1162

1163

1164

1165

1166

1167

1168

1169

1170

1171

https://hadoop.apache.org.

Jarochowska, E. (2012) 'High-resolution microtaphofacies analysis of a carbonate tidal channel and tidally influenced lagoon, pigeon creek, San Salvador Island, Bahamas', PALAIOS, 27(3), pp. 151-170. doi: 10.2110/palo.2011.p11-063r.

Jarochowska, E. et al. (2017) 'Conodonts in Silurian hypersaline environments: Specialized and unexpectedly diverse', Geology, 45(1), pp. 3-6. doi: 10.1130/G38492.1.

Kline, P. (1979) Psychometrics and Psychology. London: Academic Press.

Klubi, E. et al. (2018) 'Impact of gold-mining activity on trace elements enrichment in the West African estuaries: The case of Pra and Ankobra rivers with the Volta estuary (Ghana) as the reference', Journal of Geochemical Exploration, 190, pp. 229-244. doi: 10.1016/j.gexplo.2018.03.014.

Koutsodendris, A. et al. (2020) 'A Plio-Pleistocene (c. 0-4 Ma) cyclostratigraphy for IODP Site U1478 (Mozambique Channel, SW Indian Ocean): Exploring an offshore record of paleoclimate and ecosystem variability in SE Africa', Newsletters on Stratigraphy. doi: 10.1127/nos/2020/0608.

Kowalewski, M. and Novack-Gottshall, P. (2010) 'Resampling Methods in Paleontology', The Paleontological Society Papers, 16, pp. 19-54. doi: 10.1017/S1089332600001807.

Kruskal, J. B. (1964) 'Nonmetric multidimensional scaling: a numerical method', Psychometrika, 29(2), pp. 115129.

Lanci, L. et al. (2001) 'A record of Holocene climate in the mineral magnetic record of Alpine lakes: Sägistalsee and Hinterburgsee', Earth and Planetary Science Letters, 188(1-2), pp. 29-44. doi: 10.1016/S0012821X(01)00301-6.

Legendre, P. and Gallagher, E. D. (2001) 'Ecologically meaningful transformations for ordination of species data', Oecologia, 129(2), pp. 271-280. doi: 10.1007/s004420100716.

Legendre, P. and Legendre, L. (2012) 'Ordination in reduced space', Developments in Environmental Modelling, 24, pp. 425-520. doi: 10.1016/B978-0-444-53868-0.50009-5.

Matlab (2020) MATLAB and Statistics Toolbox. MathWorks. Available at: https://www.mathworks.com/products/matlab.html.

Minchin, P. R. (1987) 'An evaluation of the relative robustness of techniques for ecological ordination', in Prentice, I. C. and van der Maarel, E. (eds) Theory and models in vegetation science. Dordrecht: Advances in vegetation science, vol 8., Springer, pp. 89-107. doi: 10.1007/978-94-009-4061-1_9.

More, K. D. et al. (2018) 'A 43 kyr record of protist communities and their response to oxygen minimum zone variability in the Northeastern Arabian Sea', Earth and Planetary Science Letters, 496, pp. 248-256. doi: 10.1016/j.epsl.2018.05.045.

Mulaik, S. A. (1985) 'Exploratory Statistics and Empiricism', Philosophy of Science, 52(3), pp. 410-430. doi: $10.1086 / 289258$.

Neuwirth, E. (2014) RColorBrewer: ColorBrewer Palettes. $R$ package. Available at: https://CRAN.Rproject.org/package=RColorBrewer.

Nissen, S. B. et al. (2016) 'Research: Publication bias and the canonization of false facts', eLife, 5, p. e21451. doi: 10.7554/eLife.21451.001.

Oksanen, J. et al. (2019) vegan: Community Ecology Package. Available at: https://cran.rproject.org/web/packages/vegan/index.html.

Olson, C. L. (1974) 'Comparative Robustness of Six Tests in Multivariate Analysis of Variance', Journal of the American Statistical Association, 69(348), pp. 894-908. doi: 10.1080/01621459.1974.10480224.

Patzkowsky, M. E. and Holland, S. M. (2012) Understanding the distribution of fossil taxa in time and space. Chicago, USA: University of Chicago Press. Available at: https://press.uchicago.edu/ucp/books/book/chicago/S/bo12541329.html.

Piper, D. Z. and Bau, M. (2013) 'Normalized Rare Earth Elements in Water, Sediments, and Wine: Identifying Sources and Environmental Redox Conditions', American Journal of Analytical Chemistry, 04(10), pp. 69-83. doi: 
10.4236/ajac.2013.410A1009.

Popper, K. (1934) Logik der Forschung. Akademie Verlag.

Puri, M. L. and Sen, P. K. (1971) Nonparametric Methods in Multivariate Analysis. John Wiley and Sons.

R Core Team (2020) R: A Language and Environment for Statistical Computing, http://www.r-project.org/.

Read, W. A. and Dean, J. M. (1968) 'A quantitative study of a sequence of coal-bearing cycles in the Namurian of Central Scotland, 2', Sedimentology, 10(2), pp. 121-136. doi: 10.1111/j.1365-3091.1968.tb01104.x.

Reimann, C. et al. (2008) Statistical Data Analysis Explained. Chichester, UK: John Wiley and Sons, Ltd. doi: $10.1002 / 9780470987605$.

Rencher, A. C. (2003) Methods of Multivariate Analysis. Wiley.

Reyment, R. A. and Savazzi, E. (1999) Aspects of Multivariate Statistical Analysis in Geology. Elsevier. doi: 10.1016/B978-0-444-82568-1.X5012-5.

Romesburg, C. (2004) Cluster Analysis for Researchers. Lulu Press.

Sharma, S., Bawa, S. and Lomash, H. (2016) 'Proliferation of Social Computing: Cultural Computing Paradigm', International Journal of Computer Applications, 137(9), pp. 27-30. doi: 10.5120/ijca2016908922.

Shaukat, S. S., Rao, T. A. and Khan, M. A. (2016) 'Impact of sample size on principal component analysis ordination of an environmental data set: effects on eigenstructure', Ekológia (Bratislava), 35(2), pp. 173-190. doi: 10.1515/eko-2016-0014.

Shi, G. R. (1993) 'Multivariate data analysis in palaeoecology and palaeobiogeography-a review', Palaeogeography, Palaeoclimatology, Palaeoecology, 105(3-4), pp. 199-234. doi: 10.1016/00310182(93)90084-V.

van Son, T. C. and Halvorsen, R. (2014) 'Multiple Parallel Ordinations: the Importance of Choice of Ordination Method and Weighting of Species Abundance Data', Sommerfeltia, 37(1), pp. 1-37. doi: 10.2478/som-20140001.

Syms, C. (2008) 'Ordination', in Jørgensen, S. E. and Fath, B. D. (eds) Encyclopedia of Ecology. Elsevier, pp. 25722581. doi: 10.1016/B978-008045405-4.00524-3.

Taguchi, Y. -h. and Oono, Y. (2005) 'Relational patterns of gene expression via non-metric multidimensional scaling analysis', Bioinformatics, 21(6), pp. 730-740. doi: 10.1093/bioinformatics/bti067.

TIBCO Software (2018) Statistica.

Todorov, V. and Filzmoser, P. (2013) 'Comparing Classical and Robust Sparse PCA', in Kruse, R. et al. (eds) Synergies of Soft Computing and Statistics for Intelligent Data Analysis. Advances in Intelligent Systems and Computing, vol 190. Springer, pp. 283-291. doi: 10.1007/978-3-642-33042-1_31.

Tolosana-Delgado, R. (2012) 'Uses and misuses of compositional data in sedimentology', Sedimentary Geology, 280, pp. 60-79. doi: 10.1016/j.sedgeo.2012.05.005.

Tomašových, A. (2004) 'Microfacies and depositional environment of an Upper Triassic intra-platform carbonate basin: the Fatric Unit of the West Carpathians (Slovakia)', Facies, 50(1), pp. 77-105. doi: 10.1007/s10347-0040004-y.

Tyler, C. L. and Kowalewski, M. (2014) 'Utility of Marine Benthic Associations as a Multivariate Proxy of Paleobathymetry: A Direct Test from Recent Coastal Ecosystems of North Carolina', PLOS ONE. Edited by K. Swadling, 9(4), p. e95711. doi: 10.1371/journal.pone.0095711.

Van der Weijden, C. H. (2002) 'Pitfalls of normalization of marine geochemical data using a common divisor', Marine Geology, 184(3-4), pp. 167-187. doi: 10.1016/S0025-3227(01)00297-3.

van de Velden, M., lodice D’Enza, A. and Markos, A. (2019) 'Distance-based clustering of mixed data', Wiley Interdisciplinary Reviews: Computational Statistics, 11(3), p. e1456. doi: 10.1002/wics.1456.

$\mathrm{Vu}, \mathrm{V}$. Q. (2011) ggbiplot: A ggplot2 based biplot. $R$ package version 0.55 . Available at: http://github.com/vqv/ggbiplot. 
1217 Wackernagel, H. (2003) Multivariate Geostatistics. Berlin, Heidelberg: Springer Berlin Heidelberg. doi: 1218 10.1007/978-3-662-05294-5.

1219 Warne, R. T. et al. (2012) 'Statistical Methods Used in Gifted Education Journals, 2006-2010', Gifted Child 1220 Quarterly, 56(3), pp. 134-149. doi: 10.1177/0016986212444122.

1221 Wartenberg, D., Ferson, S. and Rohlf, F. J. (1987) 'Putting Things in Order: A Critique of Detrended 1222 Correspondence Analysis', The American Naturalist, 129(3), pp. 434-448. doi: 10.1086/284647.

1223 Whittaker, R. H. and Gauch, H. G. (1978) 'Evaluation of Ordination Techniques', in Whittaker, R.H. (ed.) 1224 Ordination of Plant Communities. Dordrecht: Springer, pp. 277-336. doi: 10.1007/978-94-009-7989-5_10.

1225 Wickham, H. (2016) ggplot2: Elegant Graphics for Data Analysis. New York, NY: Springer. doi: 10.1007/978-31226 319-24277-4.

1227 Wildi, O. (2018) 'Evaluating the Predictive Power of Ordination Methods in Ecological Context', Mathematics, 1228 6(12), p. 295. doi: 10.3390/math6120295.

1229 Wilkinson, M. D. et al. (2016) 'The FAIR Guiding Principles for scientific data management and stewardship', 1230 Scientific Data, 3(1), p. 160018. doi: 10.1038/sdata.2016.18.

1231 Zar, J. H. (2010) Biostatistical Analysis. Pearson.

1232 Zuschin, M., Harzhauser, M. and Mandic, O. (2007) 'The stratigraphic and sedimentologic framework of fine1233 scale faunal replacements in the middle Miocene of the Vienna Basin (Austria)', PALAIOS, 22(3), pp. 285-295. doi: 10.2110/palo.2005.p05-023r. 
1236 Table 1: summary description of the properties of PCA, NMDS and DCA

\begin{tabular}{|c|c|c|c|}
\hline & PCA & NMDS & DCA \\
\hline $\begin{array}{l}\text { Brief description of } \\
\text { operation }\end{array}$ & $\begin{array}{l}\text { Orthogonal combination } \\
\text { of } n \text {-dimensional } \\
\text { variables }\end{array}$ & $\begin{array}{l}\text { Lower dimensional } \\
\text { optimization of } \\
\text { dissimilarity between } \\
\text { data points }\end{array}$ & $\begin{array}{l}\text { Consequantive } \\
\text { dimensional warping of } n \text { - } \\
\text { dimensional variables }\end{array}$ \\
\hline $\begin{array}{l}\text { Measurement of } \\
\text { dimensional optimization }\end{array}$ & Variance & Stress & Correspondence \\
\hline Variables & $\begin{array}{l}\text { Quantitative data, linear } \\
\text { relationships }\end{array}$ & $\begin{array}{l}\text { Quantitative, } \\
\text { semiquantitative, } \\
\text { qualitative, or mixed }\end{array}$ & $\begin{array}{l}\text { Non-negative, on a } 0 \text { to } 1 \\
\text { range, quantitative or } \\
\text { binary data }\end{array}$ \\
\hline $\begin{array}{l}\text { Assumes symmetrical } \\
\text { distribution }\end{array}$ & Yes & No & No \\
\hline Requires pre-treatments & $\begin{array}{l}\text { Sometimes (normalizing } \\
\text { transformation) }\end{array}$ & $\begin{array}{l}\text { Usually no, but this } \\
\text { depends on the similarity } \\
\text { index used }\end{array}$ & $\begin{array}{l}\text { Sometimes } \\
\text { (standardization to }[0,1] \\
\text { range) }\end{array}$ \\
\hline Consistent between runs & Yes & No & Yes \\
\hline $\begin{array}{l}\text { Preserves original } \\
\text { dimensionality } \\
\text { information }\end{array}$ & Yes & No & Yes \\
\hline $\begin{array}{l}\text { Relations between } \\
\text { variables }\end{array}$ & $\begin{array}{l}\text { Axis are a composite of } \\
\text { the vectors, vectors can } \\
\text { be represented by } \\
\text { eigenvalues }\end{array}$ & $\begin{array}{l}\text { Axis are a qualitative } \\
\text { representation of the total } \\
\text { effect }\end{array}$ & $\begin{array}{l}\text { Axis are a qualitative } \\
\text { representation of the total } \\
\text { effect but vectors can still } \\
\text { be represented in this } \\
\text { space }\end{array}$ \\
\hline
\end{tabular}


1240 Table 2: examples of compositional and non-compositional data types

\begin{tabular}{|l|l|}
\hline Compositional & Non-compositional \\
\hline Concentrations (\%, \%o, ppm, M, m etc.) & Instrumental raw counts \\
\hline Fraction of area & Absolut area units (e.g. $\left.\mathrm{m}^{2}\right)$ \\
\hline No. of counts when total sum is constant & $\begin{array}{l}\text { Morphometric measurements (length, } \\
\text { angel, diameter, No. of warts etc.) }\end{array}$ \\
\hline Relative abundances & \\
\hline
\end{tabular}

